ARTICLE

https://doi.org/10.1038/s41467-019-11911-5

\title{
Genetic programming of macrophages to perform anti-tumor functions using targeted mRNA nanocarriers
}

\author{
F. Zhang ${ }^{1}$, N.N. Parayath', C.I. Ene ${ }^{2}$, S.B. Stephan', A.L. Koehne ${ }^{3}$, M.E. Coon', E.C. Holland ${ }^{2,4,5}$ \& \\ M.T. Stephan (10) $1,6,7$
}

Tumor-associated macrophages (TAMs) usually express an M2 phenotype, which enables them to perform immunosuppressive and tumor-promoting functions. Reprogramming these TAMs toward an M1 phenotype could thwart their pro-cancer activities and unleash antitumor immunity, but efforts to accomplish this are nonspecific and elicit systemic inflammation. Here we describe a targeted nanocarrier that can deliver in vitro-transcribed mRNA encoding M1-polarizing transcription factors to reprogram TAMs without causing systemic toxicity. We demonstrate in models of ovarian cancer, melanoma, and glioblastoma that infusions of nanoparticles formulated with mRNAs encoding interferon regulatory factor 5 in combination with its activating kinase IKK $\beta$ reverse the immunosuppressive, tumorsupporting state of TAMs and reprogram them to a phenotype that induces anti-tumor immunity and promotes tumor regression. We further establish that these nanoreagents are safe for repeated dosing. Implemented in the clinic, this immunotherapy could enable physicians to obviate suppressive tumors while avoiding systemic treatments that disrupt immune homeostasis.

\footnotetext{
${ }^{1}$ Clinical Research Division, Fred Hutchinson Cancer Research Center, Seattle, WA 98109, USA. ${ }^{2}$ Department of Neurological Surgery, University of Washington School of Medicine, Seattle, WA 98195, USA. ${ }^{3}$ Comparative Pathology, Fred Hutchinson Cancer Research Center, Seattle, WA 98109, USA. ${ }^{4}$ Human Biology Division, Fred Hutchinson Cancer Research Center, Seattle, WA 98109, USA. ${ }^{5}$ Alvord Brain Tumor Center, University of Washington, Seattle, WA 98195, USA. ${ }^{6}$ Department of Bioengineering and Molecular Engineering \& Sciences Institute, University of Washington, Seattle, WA 98105, USA. ${ }^{7}$ Department of Medicine, Division of Medical Oncology, University of Washington, Seattle, WA 98195, USA. Correspondence and requests for materials should be addressed to M.T.S. (email: mstephan@fredhutch.org)
} 
$\mathrm{T}$ umor-associated macrophages (TAMs) are one of the most abundant non-neoplastic cell types found in discrete cancer locales ${ }^{1}$. Just like in healthy tissues, where macrophages have a remarkable ability for responding to environmental cues, TAMs are educated by the tumor microenvironment they experience. This produces multiple phenotypes that have a broad range of functions. TAM phenotypes can be described along a linear scale, where M1 and M2 phenotypes represent the two extremes (comparable to the $\mathrm{T}_{\mathrm{H}} 1-\mathrm{T}_{\mathrm{H}} 2$ classification) ${ }^{2,3}$. M1 macrophages are recognized as classically activated macrophages that can phagocytose pathogens. More importantly, these cells have anti-tumoral properties ${ }^{4,5}$. Unfortunately, other macrophages are polarized into the M2 phenotype. These decrease inflammation, encourage tissue repair, and provide pro-tumoral effects $^{6}$. In established progressive tumors in humans, TAMs usually express an M2-like phenotype, and thus promote tumor progression, metastasis, and resistance to chemotherapy ${ }^{7-9}$. It is therefore of key interest in cancer research to create strategies that can reprogram TAMs from a pro-tumoral (M2-like) to an antitumor (M1-like) phenotype and thereby induce immune effects that can bring about tumor regression. However, as yet there are no methods that can enable physicians to rationally and selectively reprogram TAMs for therapeutic purposes ${ }^{10}$.

For example, interleukin-12 (IL-12), IFN- $\gamma$, Toll-like receptor (TLR-) agonists, and CD40 agonists have all been reported to induce repolarization of TAMs ${ }^{11-13}$. However, these immunomodulatory agents can activate a broad range of cell types, which means they are associated with dose-limiting adverse effects and systemic toxicities ${ }^{14-16}$. Likewise, several small molecule drugs have been developed that focus on blocking the localization of TAM-precursor cells to tumors by targeting pathways involved in cell recruitment or expansion (e.g., inhibitors of CSF-1/CSF$1 \mathrm{R}^{17,18}$ or $\left.\mathrm{CCL} 2^{19}\right)$. Unfortunately, these approaches do not specifically promote macrophage anti-tumor activities and require repeated systemic exposure to large doses of the drugs. Furthermore, clinical trials of these pharmaceuticals produced low responses unless they were combined with cyto-reductive therapies or checkpoint blockade inhibitors ${ }^{19,20}$. Another factor that complicates the clinical use of CSF-1R inhibitors is the systemic depletion of normal monocytic cells, which causes high toxicity if patients are treated for prolonged periods ${ }^{21}$.

Taking advantage of the very efficient phagocytic uptake of particles by macrophages, conventional nanocarriers such as liposomes have been formulated with bisphosphonates or other anti-proliferative agents (e.g., liposomal-clodronate $)^{22}$ as a means to systemically deplete these cells, which are replaced via natural regeneration mechanisms. Also, oncolytic viruses have been used to deliver siRNA to silence immune-evasion pathways within tumors and indirectly promote phagocytosis of TAMs ${ }^{23}$. However, neither of these approaches reprograms existing macrophages with tumor-fighting capabilities.

Macrophage mannose receptor 1 (MRC1), also known as CD206, is a type I transmembrane protein that belongs to the Ctype lectin family. This receptor is expressed by macrophages and dendritic cells ${ }^{24}$. M2-like TAMs are derived from circulating monocytes that already express CD206, which is further upregulated upon extravasation of the cells at the tumor site and by exposure to factors in the perivascular tumor microenvironment $^{25}$. Because CD206 shows high expression levels in TAMs, strategies that optimize the uptake of therapeutics via these receptors have the potential to be extremely powerful. Furthermore, this directed uptake is likely to require smaller treatment doses, thereby reducing the toxicity of the delivered substances. Huhn et al., for instance, functionalized polymer nanogels as potential drug nanocarriers with a Nanobody specific for $\mathrm{CD}^{206^{26}}$, and similar concepts have been described by other groups to preferentially target TAMs while minimizing uptake by normal macrophages 27,28 .

In vitro-transcribed (IVT) mRNA has recently come into focus as a potential new drug class for delivering genetic information directly into existing cells ${ }^{29}$. These synthetic medicines can be engineered to induce the transient expression of selected proteins because they structurally resemble natural mRNA. They are easily developed, inexpensive to produce, and efficiently scalable for manufacturing purposes ${ }^{30}$. Advances in addressing the inherent challenges of this drug class, particularly related to controlling translational efficacy and immunogenicity of the IVT mRNA, have provided the basis for a broad range of potential applications ${ }^{31-33}$. In fact, clinical development of mRNA-based therapeutics has led to the formation of several university spin-off companies (for example, Argos Therapeutics, BioNTech, CureVac, eTheRNA, Ethris, Factor Bioscience, Moderna, and Onkaido), which are supported by considerable venture capital inflows to develop mRNA-based cancer immunotherapies and infectious disease vaccines ${ }^{34}$.

Here, we explore the use of IVT mRNA formulated into an injectable therapeutic that can genetically re-program TAMs into antitumor macrophages without disrupting immune homeostasis or causing systemic toxicity (Fig. 1). We find that we can condense and protect IVT mRNA, and target the genes it carries to M2-like macrophages by formulating the message into biodegradable polymeric nanoparticles (NPs) ${ }^{35}$. To provide the targets with genes encoding master regulators of macrophage polarization, we first establish that co-expression of Interferon Regulatory Factor 5 (IRF5) $^{36}$ and IKK $\beta$ (a kinase that phosphorylates and activates IRF $5^{37}$ ) imprints TAMs with a potent pro-inflammatory and cytotoxic M1 phenotype. Using in vivo test systems that faithfully model advanced-stage ovarian cancer, metastatic melanoma, and glioblastoma, we establish that serial administration of IRF5/IKK $\beta$-encoding NPs (via an intraperitoneal route for ovarian cancer, and injected intravenously to treat melanoma lung metastases or glioblastoma) substantially reduce tumor progression and, in some animals, even clear the disease. Phenotypic, functional, and gene expression studies reveal a dramatically reduced density of M2-like macrophages in tumor lesions of IRF5/IKK $\beta$ NP-treated mice compared to controls, along with increased numbers of inflammatory myeloid cells with distinct M1-type transcriptional profiles. Based on these data, our next step is to translate this technology into a clinical trial as an approach to treat ovarian cancer patients who were not responsive to other therapies. Although we will test IRF5/IKK $\beta$ NPs as a monotherapy first, our platform could ultimately reveal its full potential when used in synergy with existing immunotherapies, (e.g., $\mathrm{T}$ cell therapies, cancer vaccines, or checkpoint blockade inhibitors) by creating a therapeutic window for patients, thus stimulating a stronger overall immune response.

\section{Results}

Designing NPs to choreograph IVT mRNA transfection of TAMs. We developed a targeted mRNA delivery system that can introduce robust gene expression in the targeted cells by taking advantage of electrostatic interactions between cationic $\operatorname{poly}(\beta-$ amino ester) (PbAE) polymers and anionic mRNA (Fig. 2a). To improve the stability and translation of the mRNA encapsulated in the resulting nanocarriers, we used synthetic versions of the message that incorporate the modified ribonucleotides pseudouridine $(\Psi)^{32}$ and 5-methylcytidine $(\mathrm{m} 5 \mathrm{C})$, and that are capped with ARCA (Anti-Reverse Cap Analog) ${ }^{38}$. Quality and purity of the mRNA was confirmed before use (Supplementary Fig. 1). The mRNA is released from the mRNA-PbAE complex intracellularly by hydrolytic cleavage of ester bonds in the PbAE backbone. We 


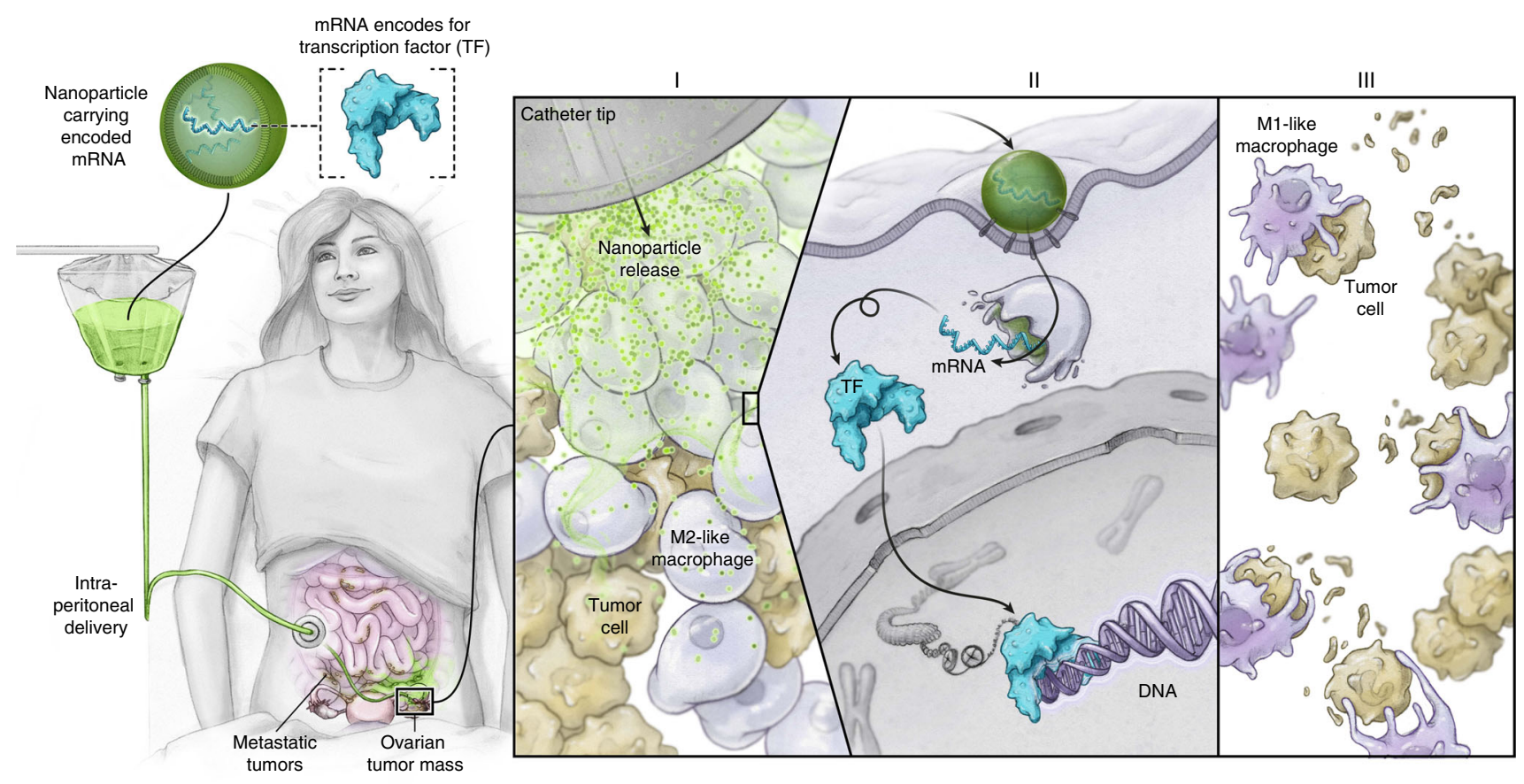

Fig. 1 Transforming tumor-associated macrophages into tumoricidal cells using mRNA nanoparticles. We developed an injectable nanocarrier delivering in vitro-transcribed mRNA encoding M1-polarizing transcription factors as a method to rationally reprogram tumor-associated macrophages (TAMs) for therapeutic purposes without causing systemic toxicity. Illustrated is the first planned clinical application, designed to treat ovarian cancer patients with repeated intraperitoneal infusions of mRNA nanoparticles

previously demonstrated efficient in vivo T cell transfection using this system ${ }^{39}$. To target the nanoparticles to TAMs as well as further stabilize the mRNA-PbAE complexes they contain, we engineered Di-mannose moieties onto their surfaces using polyglutamic acid (PGA) as a linker (Fig. 2a, Supplementary Fig. 2a). The NPs were manufactured utilizing a simple two-step, chargedriven self-assembly process. First, the synthetic mRNA was complexed with a positively-charged PBAE polymer, which condenses the mRNA into nano-sized complexes. This step was followed by the addition of PGA functionalized with Di-mannose, which shields the positive charge of the PBAE-mRNA particles and confers macrophage-targeting. The resulting mRNA nanocarriers had a size of $99.8 \pm \mathrm{SE} / 24.5 \mathrm{~nm}$ (based on 3 independently manufactured batches), a polydispersity of 0.183 , and an almost neutral surface charge $(3.40 \pm \mathrm{SE} / 2.15 \mathrm{mV} \zeta$-potential, Fig. 2b, c). We first tested the transfection efficiency of our system for murine bone marrow-derived macrophages (BMDMs) using NPs formulated with green fluorescent protein-encoding mRNA (GFP-NPs). Briefly, 50,000 BMDMs were exposed to NPs containing $1 \mu \mathrm{g}$ mRNA for $1 \mathrm{~h}$, followed by flow cytometry measurements of GFP expression the next day. Following a single NP application, we routinely transfected $31.9 \%( \pm \mathrm{SE} / 8.5 \% ; n=7)$ of these primary macrophages without reducing their viability (Fig. 2d-f). Surface modification of particles with Di-mannose was relevant, as transfection rates with untargeted (but PGAcoated) nanocarriers dropped to an average of $25 \%( \pm \mathrm{SE} / 2.1 \%$; $n=7$ ) in this inherently phagocytic cell type.

Programming immunosuppressive macrophages into proinflammatory phenotypes. To induce macrophage polarization, we next selected two mRNAs for inclusion into the NPs: the first encodes IRF5, a key member of the interferon regulatory factor family that favors the polarization of macrophages toward the M1 phenotype $^{36}$; the second encodes IKK $\beta$, a kinase that phosphorylates and activates IRF $5^{37}$. We used a ratio of 3 IRF5 mRNAs to
1 IKK $\beta$ mRNA. Using real-time quantitative PCR specific for the NP-delivered (and codon-optimized) IRF5 mRNA, we found that mRNA expression in macrophages was maximal at day 1 , resulting in a 1,500-fold increase in IRF5 relative to endogenous factor levels (Fig. 2g). As expected, gene expression was transient but IRF5 levels remained strongly upregulated through day 3 (581-fold increased) and day 5 (87-fold increased) before returning to baseline. IRF5 protein levels measured by ELISA showed a similar kinetic profile (Fig. $2 \mathrm{~h}$ ).

To determine if IRF5/IKK $\beta$-encoding NPs can reprogram M2 macrophages into the therapeutically desirable anti-cancer M1 phenotype, we used NanoString gene expression analysis. BMDMs were first cultured in the presence of interleukin-4 (IL-4) to induce a suppressive M2 phenotype (Fig. 3a). Following transfection with either control GFP-mRNA nanoparticles or IRF5/IKK $\beta$ mRNA-containing NPs, gene expression profiles were analyzed and compared with inflammatory macrophages, which we generated separately by exposing BMDMs to the TLR4 agonist Monophosphoryl Lipid A. We found that, despite being cultured in suppressive IL-4-containing medium, macrophages transfected with IRF5/IKK $\beta$ mRNA NPs display gene expression profiles similar to inflammatory macrophages (Fig. 3b). Signature M2 macrophage genes, such as Serpinb2 and Ccl11, were strongly downregulated while key M1 differentiation genes, such as Ccl5, were upregulated (Fig. 3c, d). These data establish that NPmediated expression of IRF5 and its kinase skews suppressive macrophages toward a proinflammatory phenotype.

Therapeutic effects of NP-delivered pro-M1 genes for disseminated ovarian cancer. To evaluate this treatment approach in a clinically-relevant in vivo test system, we used a model that recapitulates late-stage, unresectable ovarian tumors in C57BL/6 mice; these animals are injected with ID8 ovarian cancer cells which were tagged with luciferase to enable serial bioluminescent imaging of tumor growth. The tumors were allowed to 
a

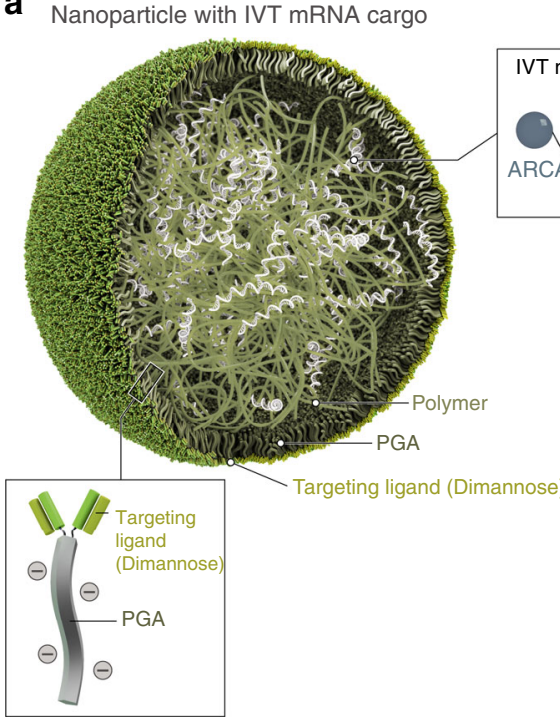

d

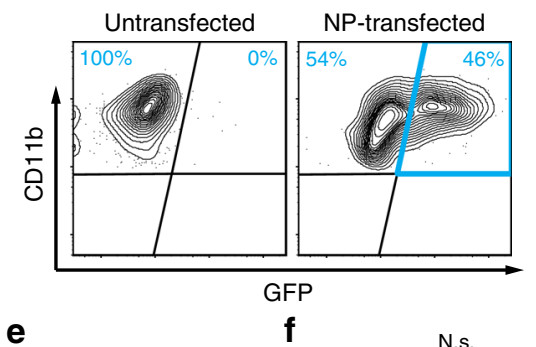

f

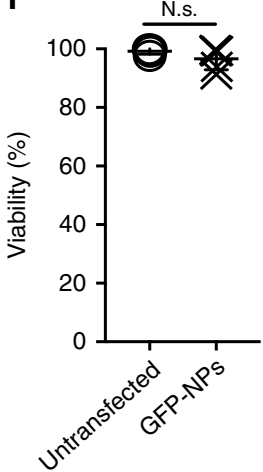

b

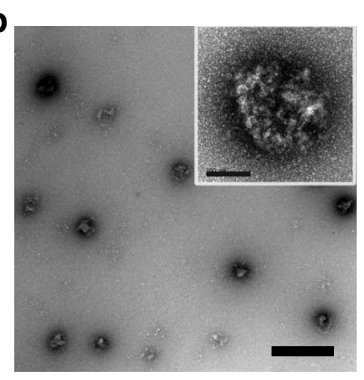

C

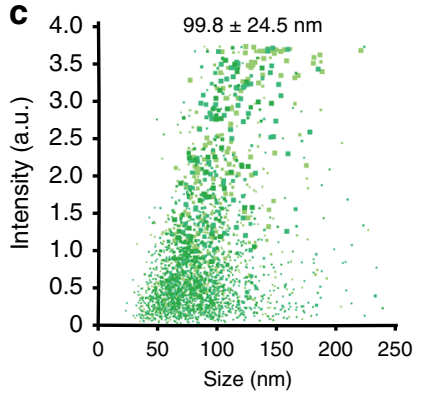

Transcription factor controlling macrophage phenotype

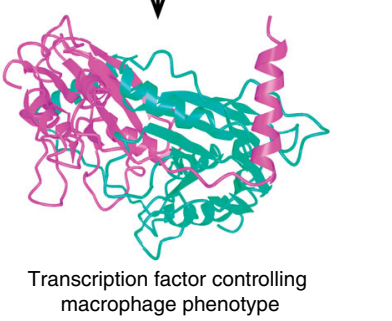

g
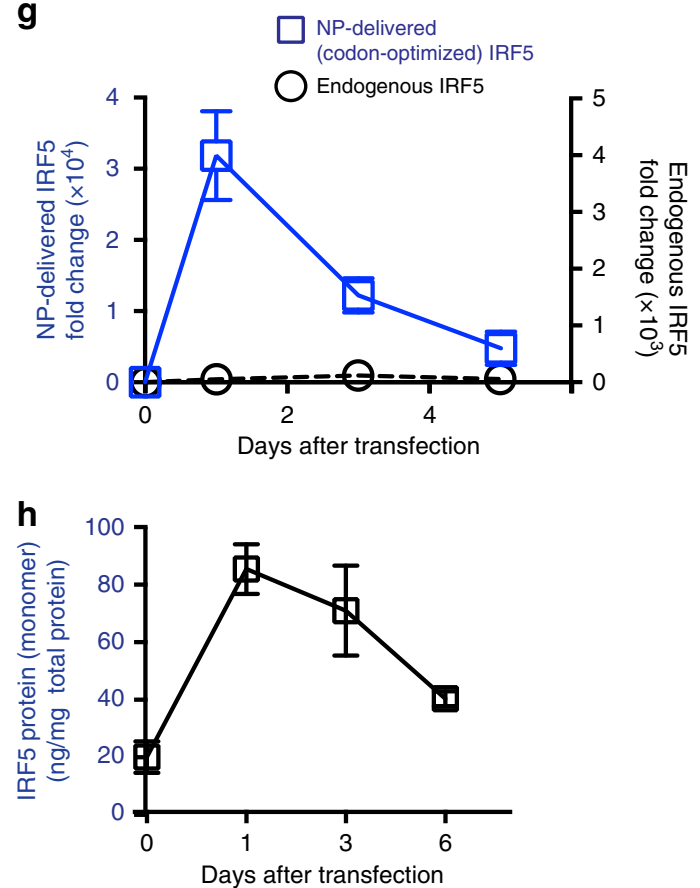

Fig. 2 Mannose receptor-targeted mRNA nanoparticles efficiently transfect M2 macrophages. a Design of macrophage-targeted polymeric NPs formulated with mRNAs encoding key regulators of macrophage polarization. The particles consist of a PbAE-mRNA polyplex core coated with a layer of PGA-Dimannose, which targets the particles to mannose receptors (CD206) expressed by M2-like macrophages. Also depicted is the synthetic mRNA encapsulated in the NP, which is engineered to encode the reprogramming transcription factors. $\mathbf{b}$ Transmission electron microscopy of a population of NPs (scale bar $200 \mathrm{~nm}$ ) and a single NP (inset, scale bar $50 \mathrm{~nm}$ ). c Size distributions, measured using a NanoSight NS300 instrument. d, e Gene-transfer efficiencies into bone marrow-derived macrophages measured by flow cytometry $24 \mathrm{~h}$ after nanoparticle transfection. $N=5$ biologically independent samples. $\mathbf{f}$ Relative viability of NP-transfected and untransfected macrophages (assessed by staining with Annexin $\mathrm{V}$ and PI). N.S., non-significant. N $=5$ biologically independent samples. $\mathbf{g}$ Expression kinetics of codon-optimized IRF5 mRNA (blue, left $Y$ axis) and endogenous IRF5 mRNA (black, right $Y$ axis) measured by qRT-PCR, $n=3$ biologically independent samples for each time point. Shown are mean values \pm SD. $\mathbf{h}$ Serial quantitative ELISA measurements of IRF5 protein (mean values $\pm S D, n=3$ )

establish for two weeks. By this stage, the mice have developed nodules throughout the peritoneal wall and in the intestinal mesentery. The animals were divided into three groups that received PBS (control), GFP-NPs (sham), or IRF5/IKK $\beta$ NP treatment at an i.p. dose of $100 \mu \mathrm{g} \mathrm{mRNA} / \mathrm{mouse} /$ week for 9 weeks (Fig. 4a). We observed that in the IRF5/IKK $\beta$ NP treated group, the disease regressed and was eventually cleared in $40 \%$ of animals (overall $142 \mathrm{~d}$ median survival versus $60 \mathrm{~d}$ in controls; Fig. 4b, c). To understand the underlying mechanisms of IRF5/ IKK $\beta$ NP-mediated anti-tumor effects, we first examined how exclusively mannose receptor-targeting confined NP interaction to phagocytes. Flow cytometry of peritoneal lavage fluid collected $24 \mathrm{~h}$ after one dose of NPs established that Di-mannose coated nanocarriers preferentially transfect 
a

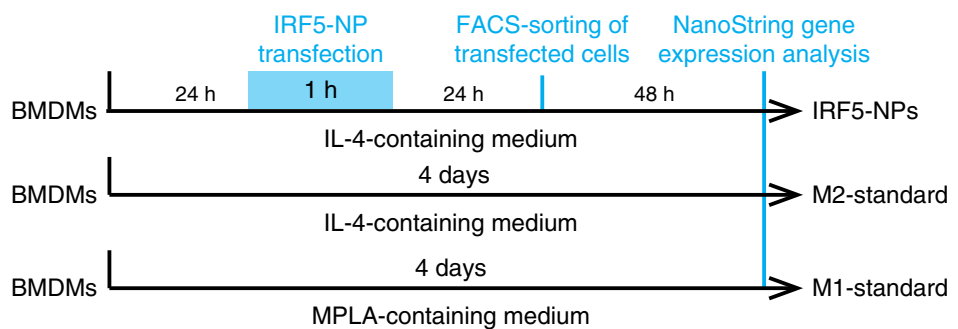

b

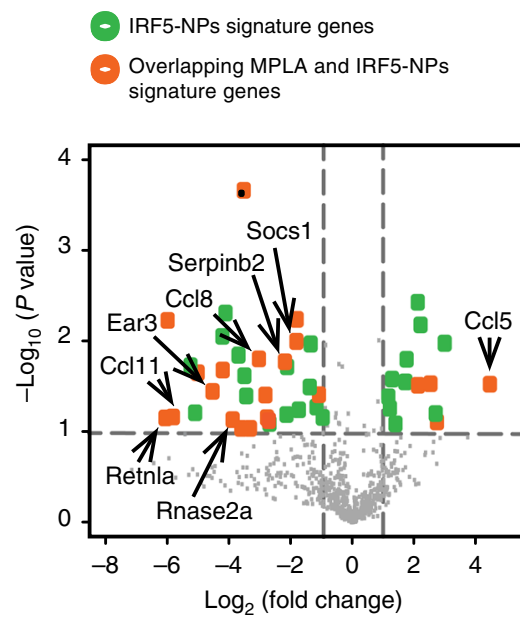

IRF5-NPs signature genes

Overlapping MPLA and IRF5-NPs signature genes

d

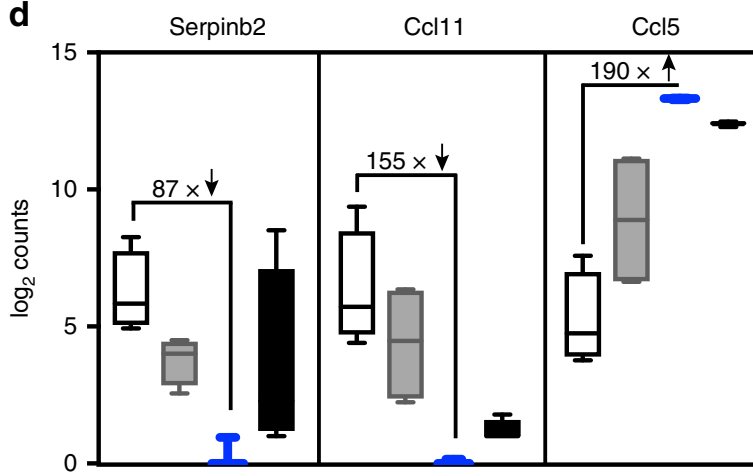

C
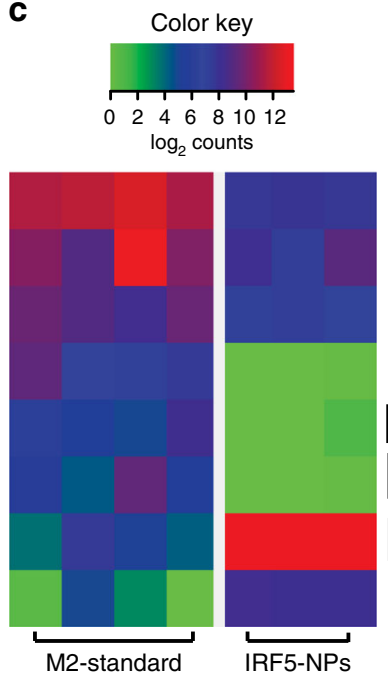

Retnla

Rnase2a

Ccl8

Ear3

Serpinb2

Col11

Ccl5

Socs 1
IL-4 (M2-standard)
GFP-control NPs
IRF5-NPs
MPLA (M1-standard)

Fig. 3 IRF5/ IKK $\beta$ mRNA nanoparticles can imprint a pro-inflammatory M1-like phenotype. a Timelines depicting NP transfection protocols and culture conditions for the BMDMs used in panels $(\mathbf{b}-\mathbf{d})$. b Gene expression profiles of IRF5/IKK $\beta$ NP-transfected macrophages compared to signature M1 cells stimulated with the Toll-like Receptor 6 agonist MPLA. Results are depicted as a Volcano plot that shows the distribution of the fold changes in gene expression. M1 signature genes are indicated. The $P$ value of overlap between IRF5/IKK $\beta$ NP-transfected macrophages and the M1 signature gene set was determined by GSEA. c Heat map of M1 signature gene expression in macrophages cultured in IL-4 versus cells cultured in IL-4 and transfected with IRF5/ IKK $\beta$ NPs. $\mathbf{d}$ Box plots showing counts for indicated genes. The boxes represent the mean values and the line in the box represents median. The bars across the boxes show the minimum and maximum values. Whiskers represent $95 \%$ confidence intervals. $N=5$ biologically independent samples

macrophages and monocytes, but also at low levels dendritic cells and neutrophils (all cell types with described CD206 expression, (Fig. 4d)). In contrast, gene transfer into CD206negative cells, such as NK cells or T cells, was undetectable. We next conducted a detailed phenotypic and functional analysis of macrophage/monocyte populations in the peritoneum of mice with established ovarian cancer following treatment with IRF5/ IKK $\beta$ nanoparticles or PBS over a 3-week period (two weekly injections). Flow cytometric analysis revealed that IRF5/IKK $\beta$ NPs reduced the population of immune-suppressive macrophages (Ly6C-, F4/80+, CD206+) to an average 2.6\% $\pm \mathrm{SE} / 2.1 \%$ $(n=5)$ versus $43 \% \pm \mathrm{SE} / 15.6 \%$ in controls (Fig. $4 \mathrm{e}$, f; Supplementary Fig. 3). Conversely, the fraction of M1-like macrophages increased from $0.5 \% \pm \mathrm{SE} / 0.2 \%$ to $10.2 \% \pm \mathrm{SE} / 4.1 \%$ (Fig. 4e, g). IRF5 gene therapy also affected the population of other immune cells. In particular, inflammatory monocytes (CD11b+, Ly6C+, Ly6G-) were more abundant $(73.4 \% \pm \mathrm{SE} /$ $3.6 \%$ compared to $4.5 \% \pm \mathrm{SE} / 1.9 \%$ in untreated mice). One interesting finding in all IRF5 NP-treated animals was multifocal dense clusters of lymphocytes present within or surrounding the neoplasms (Fig. 4h), indicating that genetic programming of immune-stimulatory macrophages may restore lymphocyte migration and infiltration into solid tumors.

We isolated peritoneal macrophages by fluorescence-activated cell sorting to analyze their cytokine secretion, and detected a robust increase in the release of pro-inflammatory (anti-tumor) 
a

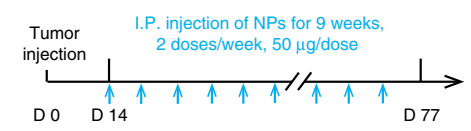

b

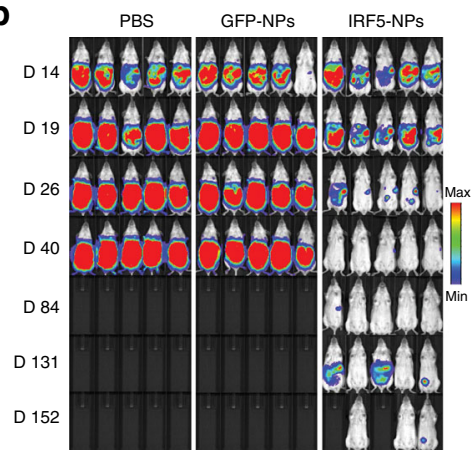

C
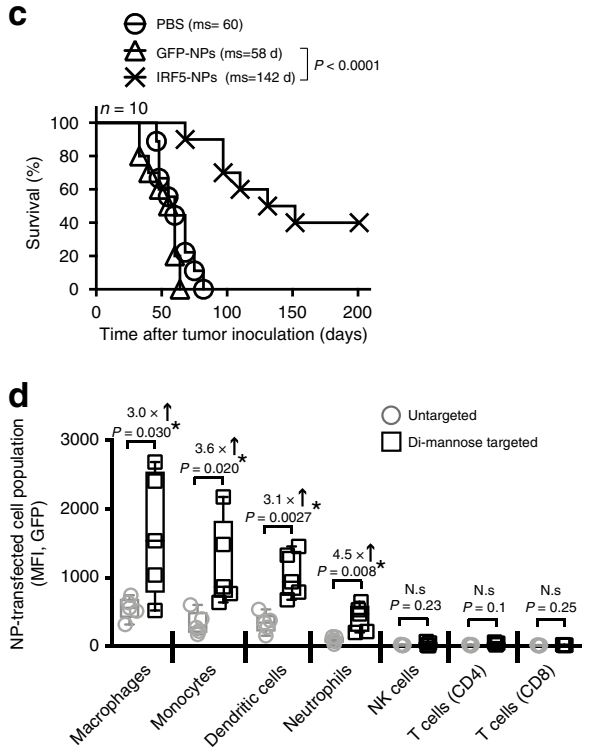

e

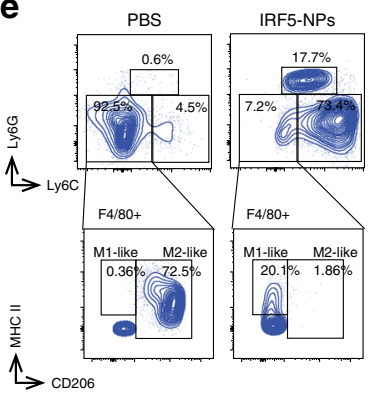

f $\quad$ M2-like (\%)

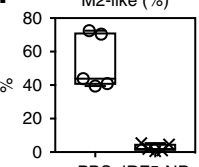

PBS IRF5-NPS

$\mathbf{g}_{25}$ M1-like (\%)

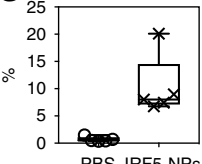

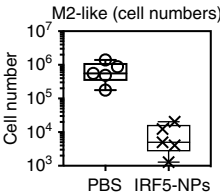

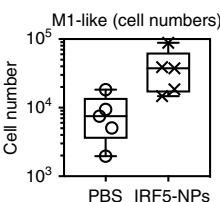

h
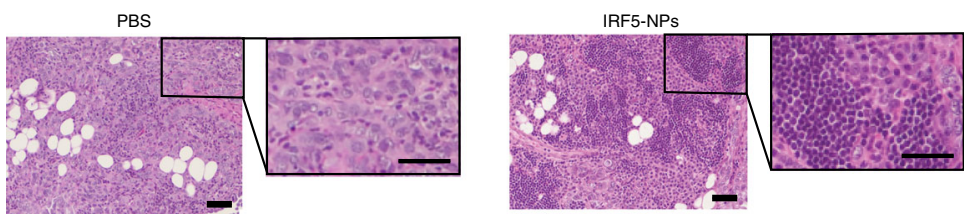

i
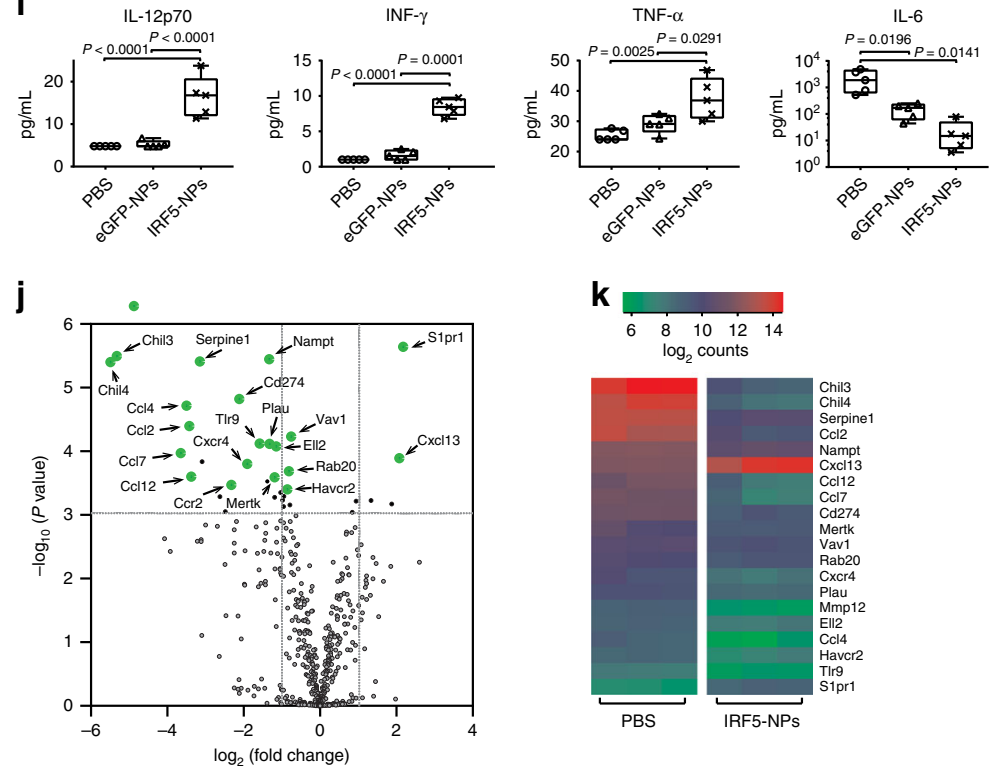

$\mathbf{K}$ $\begin{array}{lllll}6 & 8 & 10 & 12 & 14\end{array}$

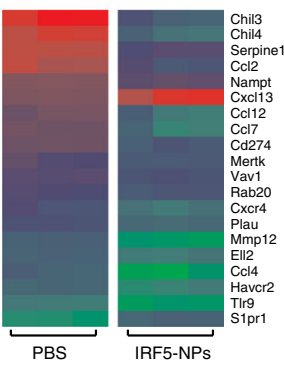

Fig. 4 IRF5/IKK $\beta$ mRNA nanoparticles double survival of mice with ovarian cancer. a Time lines and dosing regimens. b Sequential bioluminescence imaging of tumor growth. c Kaplan-Meier survival curves. Statistical analysis was performed using the log-rank test. $N=10$ biologically independent animals. d Flow cytometric quantitation of in vivo transfection rates in different immune cell subpopulations $48 \mathrm{~h}$ after a single i.p. dose of untargeted versus Di-mannose-coated NPs carrying GFP mRNA: macrophages (CD45+, CD11b+, MHCll+, CD11c-, Ly6C-/low, Ly6G-), monocytes (CD45+, CD11b+, MHCll+, CD11c-, Ly6C+, Ly6G-), dendritic cells (CD45+, CD11c+, CD11b-, MHCll+), neutrophils (CD45+, CD11b+, MHCII+, CD11c-, Ly6G+), CD4+ T cells (CD45+, TCR- $\beta$ chain,+ CD4+, CD8-), CD8+ T cells (CD45+, TCR- $\beta$ chain,+ CD4-, CD8+), and natural killer cells (CD45+, TCR- $\beta$ chain-, $\mathrm{CD} 49 \mathrm{~b}+$ ) were measured. e Flow cytometric analysis of macrophage phenotypes in the peritoneum of mice with disseminated ID8 ovarian cancer. Animals were either treated with 4 doses of IRF5/IKK $\beta$ NPs or PBS. $\mathbf{f}$ Box plots summarizing relative percent (left panel) and absolute numbers (right panel) of Ly6C-, F4/80+, and CD206+ (M2-like) macrophages. Corresponding numbers for Ly6C-, F4/80+, and CD206- (M1-like) macrophages are shown in ( $\mathbf{g}$ ). $\mathbf{h}$ Representative hematoxylin and eosin (H\&E)-stained sections of ovarian tumor-infiltrated mesenteries isolated from PBS controls (left panel) or IRF5/IKK $\beta$ NP-treated animals (right panel; scale bar $100 \mu \mathrm{m}$ ). Tenfold magnifications of representative malignant lesions are shown on the right (scale bar $50 \mu \mathrm{m}$ ). i Luminex assay measuring cytokines produced by isolated peritoneal macrophages from each treatment group. In parallel experiments, FACS-sorted CD11b+, F4/80+ peritoneal macrophages were directly analyzed by NanoString gene expression analysis. j Results are depicted as a Volcano plot. k Heat map of signature gene expression in macrophages isolated from mice treated with IRF5-NPs versus control PBS. All boxes in boxplots in this figure represent the mean values and the line in the box represents median. The bars across the boxes show the minimum and maximum values. Whiskers represent $95 \%$ confidence intervals. $N=5$ biologically independent samples

cytokines IL-12 (3.4-fold higher), IFN- $\gamma$ (8.4-fold higher), and TNF-a (1.5-fold higher), whereas the levels of IL-6, a regulatory cytokine associated with differentiation toward alternatively activated (M2-like) macrophages, were reduced by 97-fold; Fig. 4i). Genome expression profiling confirmed differentiation toward an M1-like macrophage phenotype in IRF5/IKK $\beta$ nanoparticle-treated mice (Fig. 4j, k; Supplementary Fig. 4).
Role of host $\mathbf{T}$ cells. To study in more detail how NP-mediated macrophage reprogramming affects the tumor immune cell composition, we phenotyped lymphocytes and myeloid-cells in mesenteric ovarian cancer lesions by confocal microscopy. We found that IRF5/IKK $\beta$ NPs increased T cell infiltration into tumors by an average 10.6-fold (CD8) and 3.5-fold (CD4; Fig. 5a, b). Also, densities of neutrophils increased by 16.2 -fold (Fig. $5 c$, d). To 
a

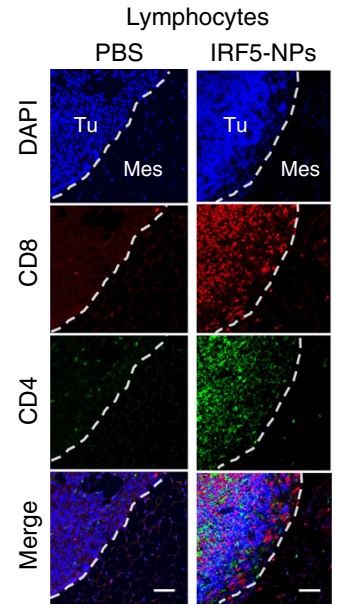

b

CD8 $+\mathrm{T}$ cells $\mathrm{CD} 4+\mathrm{T}$ cells

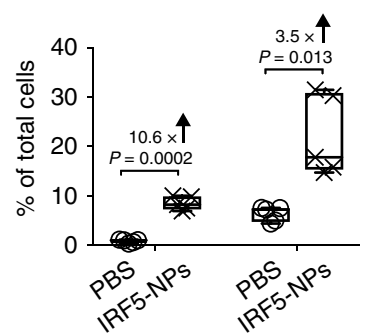

c

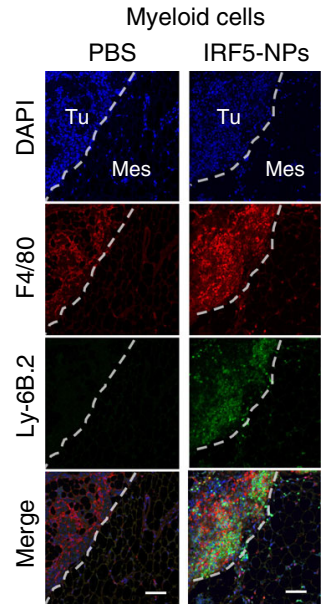

d

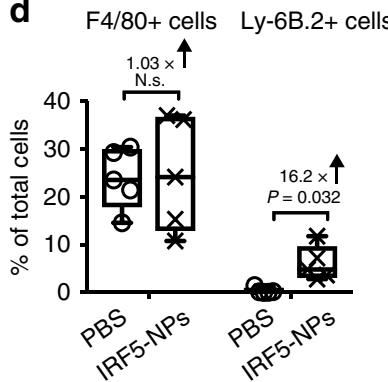

e

T cell-depletion experiment

I.P. injection of NPs for 3 weeks

Tumor inoculation 2 doses per week, $50 \mu \mathrm{g} /$ dose (mRNA base)

\begin{tabular}{|c|c|c|c|c|}
\hline$r$ inoculation & & Week 1 & Week 2 & Week 3 \\
\hline D-14 & D 01 & $\uparrow \begin{array}{lll}\uparrow & \uparrow & \mathrm{D} 7\end{array}$ & $\uparrow \uparrow \quad \uparrow \quad 14 \uparrow$ & 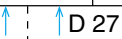 \\
\hline
\end{tabular}

f
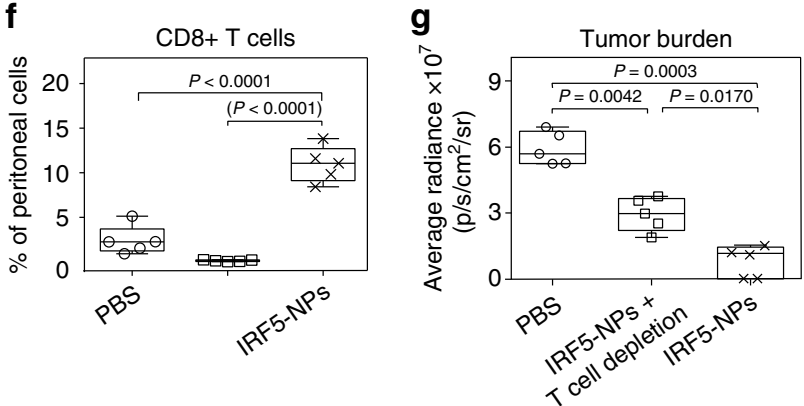

Fig. $5 \mathrm{~T}$ cells contribute to anti-tumor effects achieved with macrophage-programming nanoparticles. a-e Nanoparticle-mediated macrophage programming increases T cell recruitment into tumor lesions. Shown are representative confocal images of peritoneal metastases of ID8 ovarian cancer cells in the mesentery. Tissues were collected after 6 biweekly i.p. injections of PBS or IRF5/IKK $\beta$ NPs ( $50 \mu g$ mRNA/dose), and were stained for the indicated lymphocyte- and myeloid-markers $(\mathbf{a}, \mathbf{c})$. Tu $=$ Tumor, Mes = Mesentery. Scale bar: $100 \mu \mathrm{m}$. b, $\mathbf{d}$ Box plots showing fluorescent signals for each phenotypic marker using Halo ${ }^{\mathrm{TM}}$ image analysis software. $N=5$. The boxes represent the mean values and the line in the box represents median. The bars across the boxes show the minimum and maximum values. Whiskers represent $95 \%$ confidence intervals. $N=5$ biologically independent samples. e Experimental design for CD8 $+\mathrm{T}$ cell depletion studies in C57BL/6 mice with disseminated ID8 ovarian cancer. To deplete CD8+ T cells, mice were injected i.p. with $1 \mathrm{mg}$ anti-CD8b mAb (YTS169.4) three days before the first nanoparticle administration, followed by one $0.5 \mathrm{mg}$ dose every five days for a total of six doses. f Flow cytometry of peritoneal cells collected on day 27 established efficient depletion of CD8+ T cells (average $98.7 \%$ ). $\mathbf{g}$ Plots of ID8 tumor luciferase signal intensities after six nanoparticle injections (day 27). On each box plot, the central mark indicates the median, and the bottom and top edges of the box indicate the interquartile range. Whiskers represent $95 \%$ confidence intervals. $N=5$ biologically independent samples. Pairwise differences in photon counts between treatment groups were analyzed using the Wilcoxon rank-sum test. Shown are data for five mice per treatment condition pooled from two independent experiments

measure how much the anti-tumor responses we achieved with IRF5/IKK $\beta$ NP macrophage reprogramming were mediated by host $\mathrm{T}$ cells, we next used monoclonal antibodies to deplete CD8+ $\mathrm{T}$ cells in mice with established ID8 ovarian cancer (Fig. 5e, f). Therapeutic responses between fully immunocompetent and lymphocyte-depleted mice were compared after six IRF5/IKK $\beta$ NP doses (D27) using bioluminescence tumor imaging. In the absence of T cells, macrophage-programming IRF5/IKK $\beta$ NPs still induced $28.4 \%( \pm \mathrm{SE} / 3.5 \% ; n=5)$ of the anti-tumor activity observed in the presence of $\mathrm{T}$ cells (Fig. 5g). This indicates that $\mathrm{T}$ cells contribute to, but are not the sole mediators of, the anti-tumor effects achieved with macrophage-programming nanoparticles.

Biodistribution and safety. We next quantified the distribution of nanoparticles in various organs $24 \mathrm{~h}$ after intraperitoneal injection using RT-qPCR assays designed to detect only nanoparticle-delivered (codon optimized) IRF5. The highest concentrations of IVT mRNA were found in organs located in the peritoneum, including liver, spleen, intestine, pancreas, and diaphragm (Fig. 6a). We also detected small amounts of particledelivered mRNA in organs that lie outside of the peritoneum (heart, lungs, kidneys), suggesting that a fraction of i.p. injected nanocarriers entered the systemic circulation. Guided by the distribution data, we next assessed whether these nanoreagents are biocompatible and safe for repeated dosing. Mice were injected with a total of eight doses of IRF5/IKK $\beta$ NPs (two $50 \mu \mathrm{g}$ mRNA doses/week for 4 weeks, Fig. 6b). They were euthanized $24 \mathrm{~h}$ after the final dose, body weight was recorded, blood was collected by retro-orbital bleed for serum chemistry, and a complete gross necropsy was performed. There was no difference in body weights or body temperatures between groups (Supplementary Fig. 5). The following tissues were evaluated by a boardcertified staff pathologist: liver, spleen, mesentery, pancreas, stomach, kidney, heart, and lungs. Histopathological evaluation revealed in all cases multifocal dense clusters of lymphocytes within or surrounding tumor lesions, but no evidence of inflammation or frank necrosis was observed in tissues where neoplastic cells were not present (Fig. 6c). Also, serum chemistry of IRF5/IKK $\beta$ NP-treated mice was comparable to that of PBS controls, indicating that systemic toxicities did not occur (Fig. $6 \mathrm{~d})$. Because we detected small amounts of IRF5-mRNA systemically in our biodistribution studies, we designed parallel experiments to quantitate inflammatory cytokines in the 
a

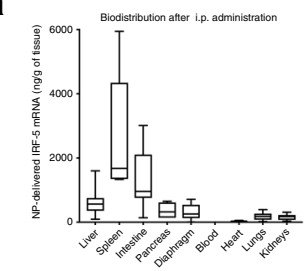

b

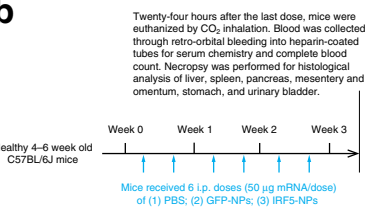

C

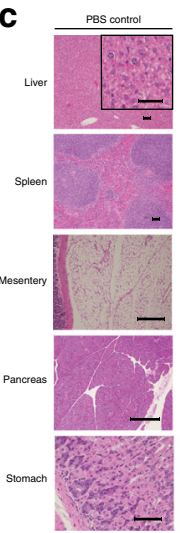

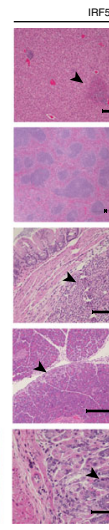
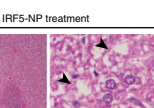

Sincose
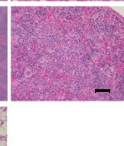$$
\text { 牲 }
$$
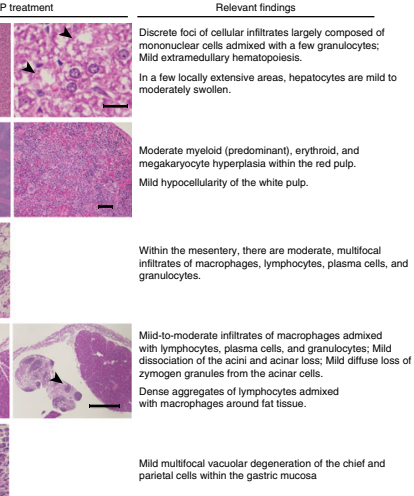

d
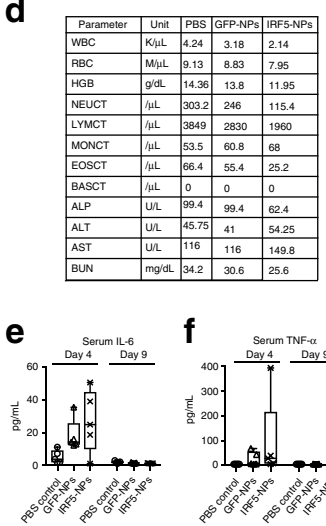

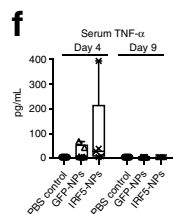

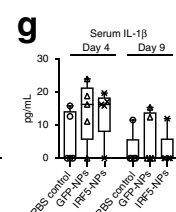

Fig. 6 Macrophage-programming mRNA nanocarriers are biocompatible and safe for repeated i.p. dosing. a In vivo biodistribution of macrophage-targeted IRF5/IKK $\beta$ NPs following i.p. administration. NP-delivered (codon optimized) mRNA was detected by qPCR $24 \mathrm{~h}$ after a single injection of particles containing $50 \mu \mathrm{g}$ mRNA. The boxes represent the mean values and the line in the box represents median. The bars across the boxes show the minimum and maximum values. Whiskers represent $95 \%$ confidence intervals. $N=19$ biologically independent animals. b-d These panels summarize a pathology report prepared by a Comparative Pathologist at FHCRC. b Schematic representation of the experimental timeline. $\mathbf{c}$ Representative H\&E-stained sections of various organs isolated from controls or NP-treated animals. Scale bar, $100 \mu \mathrm{m}$. Lesions found in the NP-treated animals are shown and described on the right. $\mathbf{d}$ Serum chemistry and blood counts. Luminex assay measurements of serum IL-6, TNF- $\alpha$, and IL-1 $\beta$ cytokines 4 or 9 days after a single i.p. injection of IRF5/IKK $\beta$ NPs are shown panels (e), (f), and (g), respectively. On each box plot, the central mark indicates the median, and the bottom and top edges of the box indicate the interquartile range. Whiskers represent $95 \%$ confidence intervals. $N=5$ biologically independent samples

peripheral blood. Following a single i.p. injection of IRF5/IKK $\beta$ NPs, we measured a moderate and transient increase in serum levels of interleukin-6 (IL-6) to an average of $26.8 \mathrm{pg} / \mathrm{mL}$ (Fig. 6e), tumor necrosis factor- $\alpha$ (TNF- $\alpha$ ) to an average of $94.7 \mathrm{pg} / \mathrm{mL}$ (Fig. 6f), and interleukin-1beta (IL-1 $\beta$ ) to an average of $14.1 \mathrm{pg} /$ $\mathrm{mL}$ (Fig. 6g). Based on previous reports, these levels are around 500 -fold lower than those associated with pathological findings and thus can be considered safe ${ }^{40,41}$.

Controlling systemic tumor metastases with intravenous infusions of IRF5/IKK $\beta$ nanoparticles. Based on the therapeutic responses we achieved with IRF5/IKK $\beta$ NPs administered directly into the peritoneal cavity to treat tumor lesions spread throughout the peritoneum, we next asked whether intravenously infused mRNA nanocarriers could program macrophages systemically to control disseminated disease. RT-qPCR biodistribution studies revealed that i.v.-infused nanocarriers preferentially deliver their mRNA cargo to organs with high levels of resident macrophages/ phagocytes, mostly the spleen, liver, and lungs (Fig. 7a). A full histopathological examination of mice after six doses of intravenously infused nanoparticles $(30 \mu \mathrm{g} / \mathrm{dose}$, Fig. $7 \mathrm{~b})$ revealed only moderate mononuclear infiltrates in the lungs and liver as well as mild red pulp expansion of the spleen due to myeloid, erythroid, and megakaryocyte hyperplasia (Fig. 7c). Importantly, overall liver function was normal in all IRF5/IKK $\beta$ NP-treated mice, with only minimally elevated blood levels of the liver enzymes alanine transaminase (ALT) and aspartate aminotransferase (AST, Fig. 7d). Serum creatinine (CRE) levels were unaltered by nanoparticle infusions, indicating normal renal function (Fig. $7 d$ ). In addition, nanoparticle treatments caused only modest increases in the expression levels of inflammatory cytokines (Fig. 7e-g). Taken together, these results indicate IRF5/IKK $\beta$ NPs are biocompatible and safe for repeated intravenous dosing. The most prominent histological lesions (mononuclear infiltrates within the parenchyma of the lung and liver) were minor reactions that typically resolve with minimal or no clinical intervention ${ }^{42}$.

To measure anti-tumor responses in a clinically relevant in vivo test system, we administered particles containing IRF5/ IKK $\beta$ mRNA into mice with disseminated pulmonary melanoma metastases (Fig. 8a). Recent work describes the foundational role of monocytes and macrophages in establishing metastases caused by this disease $\mathrm{e}^{43,44}$, and we confirmed by confocal microscopy that tumor engraftment was coordinate with phagocyte accumulation in the lungs (Fig. 8b). Tumor burdens were determined by bioluminescent imaging, and mice with detectable cancers were sorted into groups that had matching levels. Groups were then randomly assigned to treatment conditions, receiving no therapy (PBS), or intravenous injections of GFP- or IRF5/ IKK $\beta$ encapsulating nanoparticles. We found that only IRF/IKK $\beta$ nanoparticle therapy substantially reduced tumor burdens in the lungs; in fact, they improved overall survival by a mean 1.3fold (Fig. 8c, d). In parallel experiments, mice were sacrificed 22 days after tumor inoculation to validate bioluminescence tumor signals with counts of pulmonary metastases and to assess macrophage polarization by flow cytometry. The total number of metastases in the lungs of IRF5/IKK NP-treated animals was 8.7fold reduced (average $40 \pm \mathrm{SE} / 16$ metastases; $n=5$ ) compared to PBS controls (average $419 \pm$ SE/139 metastases; Fig. 8e, f). Flow cytometry of bronchoalveolar lavage fluid cells revealed a strong shift from immune-suppressive (CD206+, MHCII-, CD11c+, $\mathrm{CD} 11 \mathrm{~b}^{\text {low}}$ ) macrophages (displayed in red) toward activated (CD206-, MHCII+, CD11c-, CD11b+) phagocytes (shown in blue, Fig. $8 \mathrm{~g}, \mathrm{~h})$.

Programming tumor-suppressing phagocytes to treat glioma. For a third in vivo test system we examined glioma, which is a difficult to manage cancer type where M2-like macrophages represent the majority of non-neoplastic cells and promote tumor growth and invasion ${ }^{45}$. Currently, the standard of care for this disease is radiotherapy, which unfortunately offers only a temporary stabilization or reduction of symptoms and extends median survival by just 3 months ${ }^{46}$. To recapitulate the genetic events and subsequent molecular evolution of the disease, we used the RCAS-PDGF-B/Nestin-Tv-a; Ink4a/Arf-/-; Pten-/transgenic mouse model of PDGF $\beta$-driven glioma (PDG mice $\left.{ }^{47,48}\right)$. Brain tissue was stereotactically injected with a mixture of DF- 1 cells transfected with either RCAS-PDGF $\beta$ or RCAScre retrovirus (1:1 mixture, $2 \mu \mathrm{L})$. Overexpression of the PDGF $\beta$ oncogene and the absence of the tumor suppressor genes Ink4aarf and Pten in glioma progenitors led to the formation of 

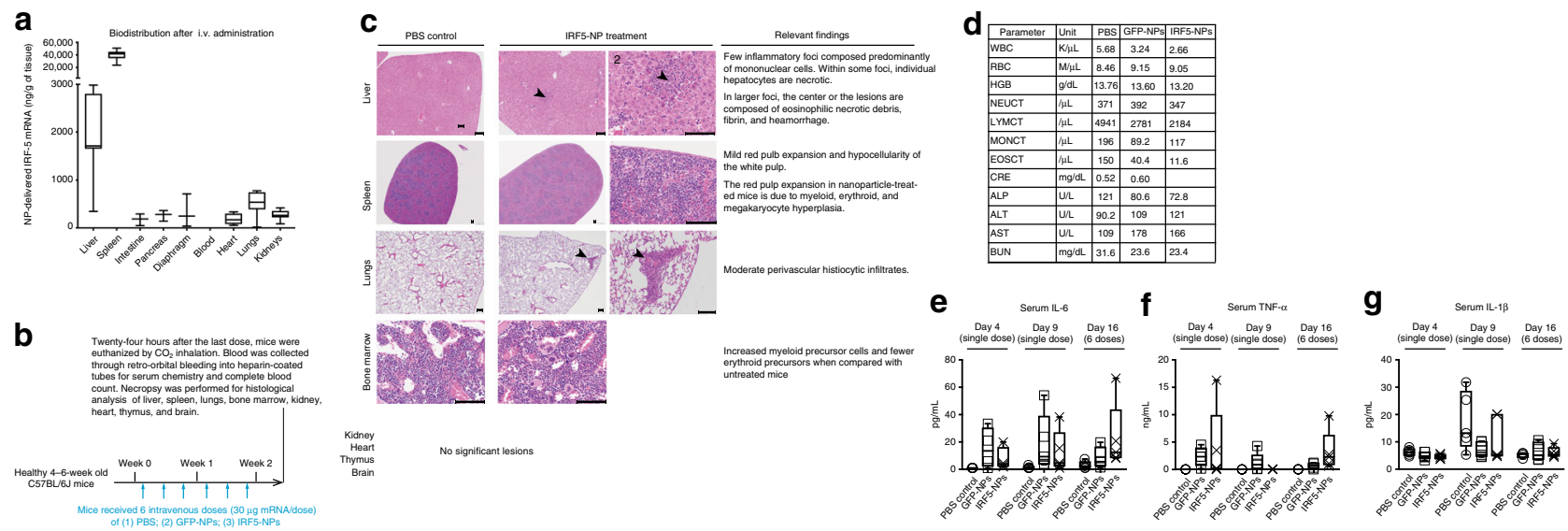

Fig. 7 Repeated intravenous infusions of nanocarriers are not associated with systemic toxicities. a In vivo biodistribution of macrophage-targeted IRF5/ IKK $\beta$ NPs following i.v. administration. Codon-optimized mRNA was measured by qPCR $24 \mathrm{~h}$ after a single i.v. injection of particles containing $50 \mu g$ mRNA. The boxes represent the mean values and the line in the box represents median. The bars across the boxes show the minimum and maximum values. Whiskers represent $95 \%$ confidence intervals. $N=19$ biologically independent animals. b-d These panels summarize a pathology report prepared by a Comparative Pathologist at FHCRC. b Schematic representation of the experimental timeline. c Representative H\&E-stained sections of various organs isolated from controls or NP-treated animals. Scale bars, $100 \mu \mathrm{m}$. Lesions found in the NP-treated animals are shown and described on the right. $\mathbf{d}$ Serum chemistry and blood counts. Luminex assay measurements of serum IL- 6 , TNF- $\alpha$, and IL-1 $\beta$ cytokines 4 or 9 days after a single i.v. injection and 2 days after 6 repeated doses (Day 16) of IRF5/IKK $\beta$ NPs are shown panels (e), (f), and (g) respectively. On each box plot, the central mark indicates the median, and the bottom and top edges of the box indicate the interquartile range. Whiskers represent $95 \%$ confidence intervals. $N=5$ biologically independent samples

4-5 mm diameter tumors (Fig. 9a) with a nearly complete penetrance within 21 days (as established previously ${ }^{47}$ ). Using immunofluorescence, we confirmed the presence of tumorinfiltrating $\left(\mathrm{CD}^{+} 8^{+}\right)$macrophages (Fig. 9b, indicated in red) in established gliomas (shown in green). Flow cytometry revealed that the $\mathrm{F} 4 / 80+, \mathrm{CD} 11 \mathrm{~b}+$ macrophage population accounted for $32.8 \%$ of total cells in the tumor, which is ninefold higher than seen in age-matched healthy control mice (3.7\%) (Fig. 9c). The PDG mice in our experiments express the firefly luciferase gene linked to a key cancer gene promoter. Bioluminescence from this reporter has been demonstrated to be positively correlated with tumor grade ${ }^{49}$, so we used it to monitor tumor development every four days after the onset of treatment. We first tested IRF/IKK $\beta$ NPs as a monotherapy: PDG mice received intravenous infusions of 9 doses of NPs loaded with IRF5/IKK $\beta$ mRNA, or PBS in the control group (3 doses/week for 3 weeks). We first found that IRF/IKK $\beta$ NP treatments only modestly suppressed tumor progression (producing on average only a 5-day survival advantage compared to untreated controls; Fig. 9d). However, combining radiotherapy as the standard-of-care with IRF5/IKK $\beta$ NP injections substantially reduced tumor growth and more than doubled the survival of treated mice compared to the PBS control group (52 days versus 25 days, respectively; Fig. 9e, f).

In conclusion, our in vivo results from three preclinical solid tumor models demonstrate that nanoparticles, administered either locally or systemically, can deliver genes encoding master regulators of macrophage polarization to re-program immunosuppressive macrophages into tumor-clearing phenotypes.

Translation from murine to human macrophages. To confirm that our data acquired in mice have relevance to treat human disease, we fabricated NPs delivering IVT mRNA encoding human IRF5 and IKK $\beta$ (huIRF5 NPs). We used the human monocytic cell line THP-1 as a well-established M1 and M2 macrophage polarization model to test these nanocarriers ${ }^{50,51}$. M2-type macrophages were generated by treating THP-1 cells with PMA and polarizing them with IL-4 and IL-13 (Fig. 10a). To confirm that huIRF5 NPs are functional and activate the IRF pathway, we transfected THP1-Lucia ${ }^{\text {TM }}$ ISG cells with nanoparticles loaded with huIRF5/IKK $\beta$ or GFP control mRNAs. THP1-Lucia ${ }^{\mathrm{TM}}$ ISG cells secrete the fluorescent Lucia reporter under the control of an IRF-inducible promoter. This composite promoter is comprised of five IFN-stimulated response elements (ISRE) fused to an ISG54 minimal promoter, which is unresponsive to activators of the NF- $\kappa$ B or AP-1 pathways. As a result, THP1-Lucia ${ }^{\text {Tm }}$ ISG cells allow the monitoring of the IRF pathway by determining the activity of the Lucia luciferase. We found that huIRF5 NPs strongly upregulated luciferase expression in M2polarized THP-1 cells, indicating that the mRNA constructs we designed are functional in human cells (Fig. 10b, c). To determine whether IRF5 pathway activation can re-program M2-polarized THP-1 cells toward an M1-like phenotype, we measured secretion of the pro-inflammatory cytokine IL- $1 \beta$ following NP transfection. Production of IL- $1 \beta$ was significantly increased in THP-1 cells transfected with huIRF5 NPs versus untransfected controls (mean 21-fold; $P<0.0001$; unpaired, two-tailed Student's $t$ test; Fig. 10d), which correlated with a robust upregulation (10.9-fold increased MFI, $P<0.0001$; unpaired, two-tailed Student's $t$ test) of the M1 macrophage cell surface marker CD80 (Fig. 10e). Based on these data, we began working with the Nanotechnology Characterization Laboratory at the National Cancer Institute to further characterize the quality and biocompatibility of our nanocarriers, according to FDA regulations for nanomedicine with the goal of submitting an IND for a first-in-human clinical trial to test with chemotherapy-resistant ovarian carcinoma patients.

\section{Discussion}

In vitro transcribed (IVT) mRNA is gaining momentum as a potential new drug class for induction of antigen-specific immunity, especially in the field of immuno-oncology 52 . IVT has also been used as a starting material for cell therapy approaches aimed at gene editing or immune interventions ${ }^{53-55}$. Here, we explored the use of IVT mRNA to reprogram TAMs as a strategy to treat cancer. We demonstrate that suppressive M2 macrophages can be genetically reconfigured in situ into tumorclearing M1 cells by nanoparticles targeted to provide them with genes encoding master regulators of macrophage polarization. 
a

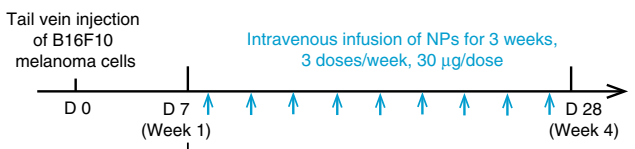

b

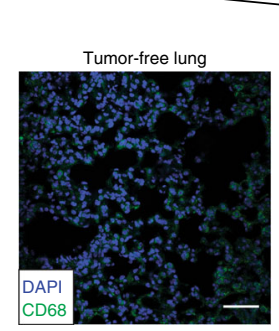

Lung with established

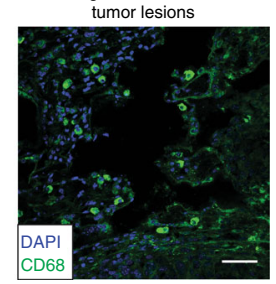

C

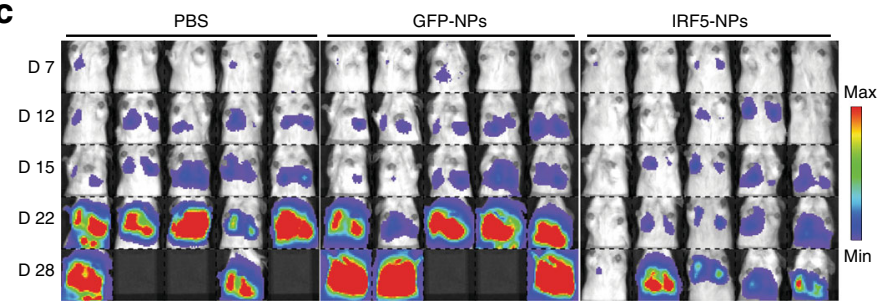

d

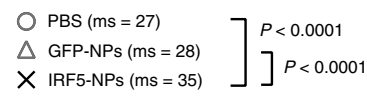
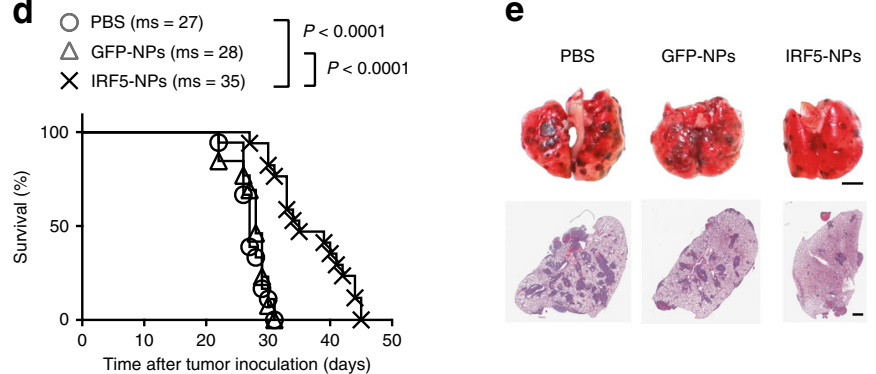

f
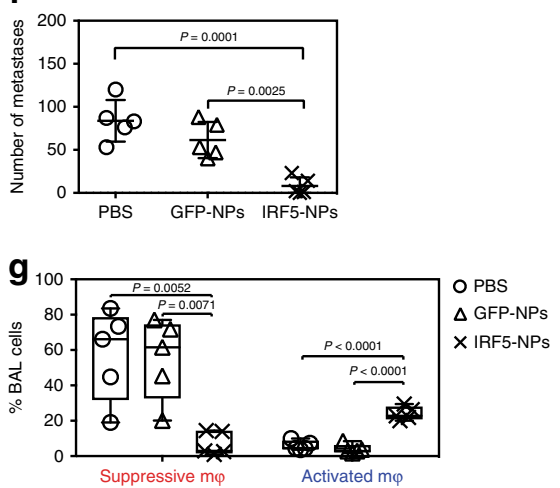
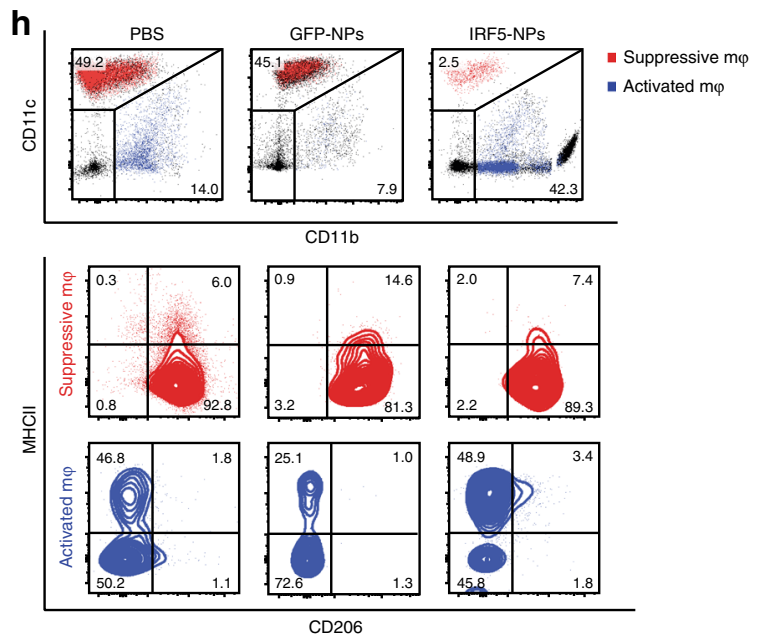

Fig. 8 Intravenously infused IRF5/IKK $\beta$ nanoparticles can control tumor metastases in the lung. a-g C57BL/6 albino mice were injected via the tail vein with $1 \times 10^{6}$ B16F10 firefly luciferase-expressing melanoma cells to establish lung metastases. After 7 days, animals were randomly assigned to either the IRF5/IKK $\beta$ NP treatment group, a control GFP NP group, or a PBS control. a Time lines and dosing regimens. b Confocal microscopy of healthy lungs (left panel) and B16F10 tumor-infiltrated lungs (right panel). Infiltrating macrophage populations fluoresce in green. c Sequential bioluminescence tumor imaging. d Kaplan-Meier survival curves for each treatment group. ms, median survival. Statistical analysis was performed using the log-rank test, and $P<0.05$ was considered significant. $N=10$ biologically independent animals. e Representative photographs (top row) and micrographs of lungs containing B16F10 melanoma metastases representing each group following 2 weeks of treatment. Counts of lung tumor foci are summarized in (f). $N=5$ biologically independent samples. $\mathbf{g}$ Phenotypic characterization of monocyte/macrophage populations in bronchoalveolar lavage from each treatment group ( $n=5$ biologically independent samples). The relative percentages of suppressive and activated macrophages are summarized by the data shown in (h)

For our proof-of-concept, we chose to express IRF5 and its activating kinase IKK $\beta$ rather than other members of the IRF protein family that can induce M1 differentiation to subsets (e.g., IRF1 or IRF8) because of the diverse impact IRF5 has on the activation of genes encoding type I interferon, inflammatory cytokines, and tumor suppressors ${ }^{36,56}$. However, our group is also interested in testing whether combinations of IRFs (e.g., IRF5 and IRF8) can synergistically improve the anti-tumor potential we describe here. Furthermore, engineered versions of IRF proteins with supra-physiological function have been reported, and we will examine these before moving the technology into clinical trials ${ }^{57,58}$. Because some IRF proteins (e.g., IRF3 and IRF4) can induce inhibitory M2 phenotypes $^{59}$, the approach we describe here could also be used to develop injectable nanomedicine configured to treat autoimmune diseases where inflammatory macrophages play key roles in the pathogenesis, such as systemic lupus erythematosus, rheumatoid arthritis, Sjogren's syndrome, multiple sclerosis, and inflammatory bowel disease ${ }^{60}$. Our platform is therefore highly adaptable and could be explored beyond cancer therapy for a wide range of disease types that are caused or facilitated by macrophages.

The structural and chemical modifications we integrated into the nanoparticle-delivered IVT mRNA constructs (depicted in Fig. 2a) have been used before to ensure intracellular stability, translational efficiency, and low immunogenicity. In particular, the incorporation of the modified nucleosides pseudouridine and 5-methylcytidine into IVT mRNA has been shown to substantially reduce immune stimulation and stabilize the molecule against RNase cleavage ${ }^{32,61}$, and these modifications have been shown to improve cytosolic persistence and protein expression ${ }^{32}$.

Immune responses generated by unmodified mRNA can be beneficial for vaccination purposes, which is currently one of the major clinical applications of mRNA drugs ${ }^{52,62}$. These selfadjuvanted vaccines are administered subcutaneously or intramuscularly, where they locally induce innate immune responses. However, for our technology, nonspecifically-induced innate immune effects comprise a detriment: in situ reprogramming of macrophages relies on systemic or intraperitoneal infusions of 
a

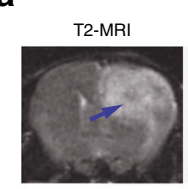

b

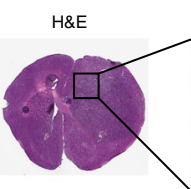

DAPI

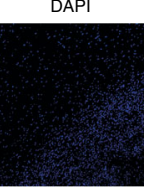

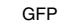

GFP
(glioma cells)

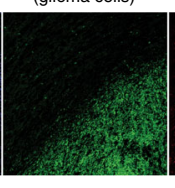

d

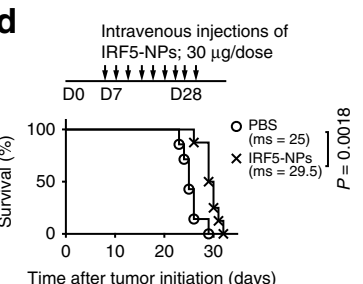

e Local brain Intravenous injections of
radiotherapy IRF5-NPs; $30 \mu \mathrm{g} /$ dose

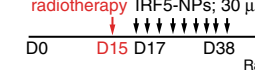

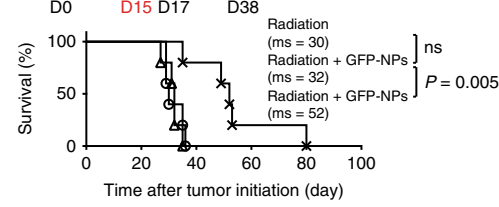

Anti-CD68 (Macrophages)
Composite

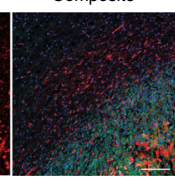

C

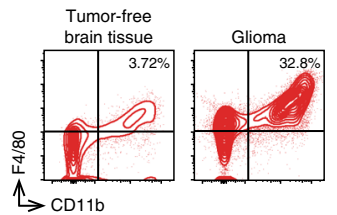

f

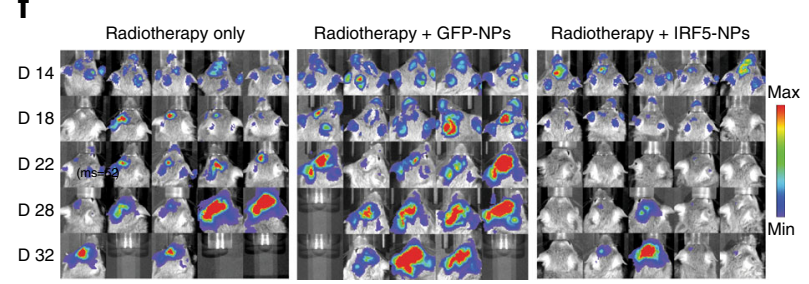

Fig. 9 Macrophage reprogramming improves the outcome of radiotherapy in glioma. a T2 MRI scan, and histological staining following initiation of a PDGF $\beta$-driven glioma in RCAS-PDGF-B/Nestin-Tv-a; Ink4a/Arf-/-; Pten-/- transgenic mice on post-induction day 21. b Confocal microscopy of CD68+ TAMs infiltrating the glioma margin. Scale bar $300 \mu \mathrm{m}$. c Flow cytometry analysis of macrophage (F4/80+, CD11b+) populations in healthy brain tissue versus glioma. d, e Kaplan-Meier survival curves of mice with established gliomas receiving IRF5/IKK $\beta$ treatments as a monotherapy (d) or combined with brain tumor radiotherapy (e). Time lines and dosing regimens are shown on top. Ms, median survival. Statistical analysis was performed using the log-rank test, and $P<0.05$ was considered statistically significant. $N=5$ biologically independent animals. $\mathbf{f}$ Sequential bioluminescence imaging of tumor progression

a

THP1-Lucia ISG (interferon-stimulated genes) express the secreted luciferase Lucia reproter gene under the control of an IRF-inducible promoter.

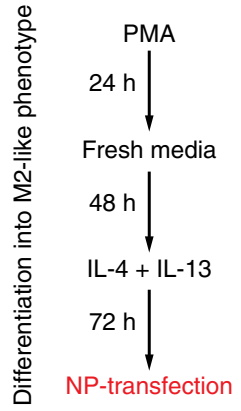

\section{b}

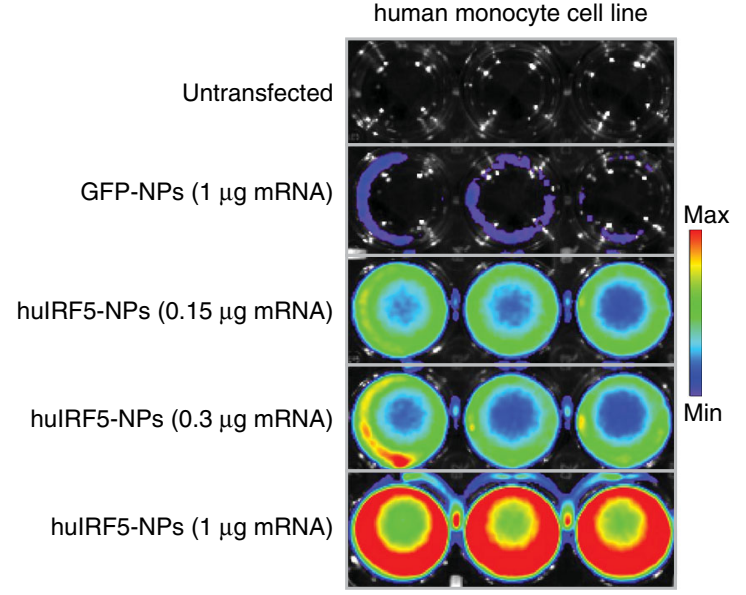

C

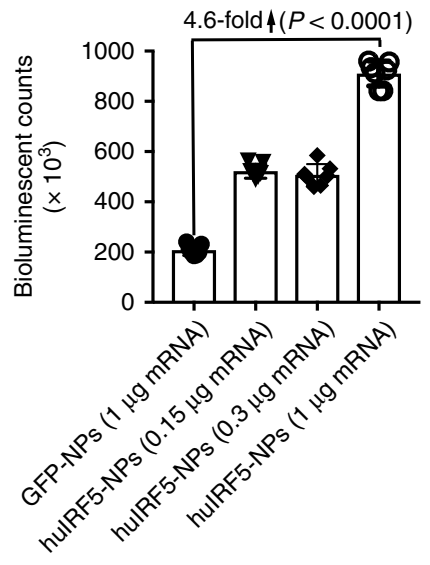

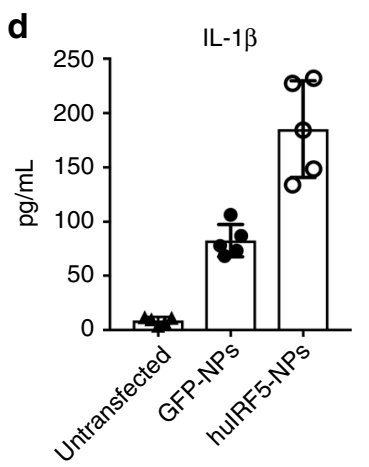
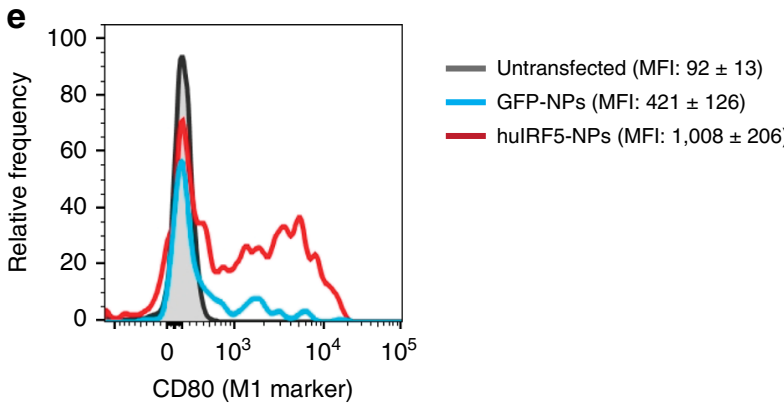

Fig. 10 MRNA-carrying nanoparticles encoding human IRF5/IKK $\beta$ reprogram human macrophages. a Time line and culture conditions to differentiate the human THP-1 monocytic cell line into suppressive M2-like macrophages. b Bioluminescent imaging of M2-differentiated THP1-Lucia cells cultured in 24well plates and transfected with indicated concentrations of NPs carrying human IRF5/ IKK $\beta$ mRNA versus control GFP mRNA. Shown are three representative wells per group. Levels of IRF-induced Lucia luciferase were determined $24 \mathrm{~h}$ after transfection using Quanti-Luc. Bioluminescent counts are summarized in the bar graph shown in (c). $N=5$ biologically independent samples. Differences in IL-1 $\beta$ cytokine secretion and surface expression of the M1-macrophage marker CD80 are shown in (d) and (e), respectively. Shown are mean values \pm SD 
mRNA therapeutics, which means unmodified mRNA constructs would cause wide-spread inflammatory toxicities and thus translational shutdown and premature degradation of the RNA. We demonstrate in Figs. 6 and 7 that IRF5/IKK $\beta$ NPs incorporating modified mRNAs are safe for repeated dosing, with only modest increases in the expression levels of inflammatory cytokines. To further improve clinical safety, the pseudouridine nucleotides used in our studies could be replaced with the recently developed N1-methyl-pseudouridine (N1m $\psi$ ) analog, which further reduces immunogenicity and at the same time improves translation of mRNA therapeutics ${ }^{63}$.

Our choice for the nanocarrier substrate was primarily guided by safety, along with compatibility with scale-up production under GMP-conditions to facilitate carrying this nanomedicine forward into human studies. Our group tested a panel of cationic materials, including hyperbranched STAR Polymer ${ }^{64}$, polyethylene glycol-grafted polyethylenimine ${ }^{65}$, and mesoporous silica nanoparticles ${ }^{66}$, and selected PBAE 447 based on its superior transfection efficacy and low biomaterial-mediated cytotoxicity in primary macrophages. The safety of PBAE 447 is related to its high biodegradability, resulting in a half-life between 1 and $7 \mathrm{~h}$ in aqueous conditions ${ }^{67}$. This time frame is ideal for mRNA gene therapy, as the polymer condenses and effectively protects nucleic acids against degradation while they are within the endosome, but releases them once the nanoparticles come in contact with the cytoplasm. These nanocarriers can be manufactured using a fast $(<10 \mathrm{~min})$ two-step, chargedriven self-assembly process. In the first step, IVT mRNA is complexed with a positively-charged PBAE polymer, which condenses the mRNA into nano-sized complexes (Fig. 2b, c). This is followed by the addition of pre-formed PGA functionalized with Di-mannose, which shields the positive charge of the PBAEmRNA particles and confers M2 macrophage-targeting. Using a fully-automated continuous-flow microfluidics system, we can now manufacture these nanodrugs with nearly uniform physical and functional characteristics at clinical scales.

At this point, it isn't difficult to predict the ways our system can extend beyond a carrier that conveys IVT mRNA across the cell membrane of macrophages. For example, we know from our therapeutic studies that administration of control nanoparticles delivering mRNA encoding GFP instead of IRF5/IKK $\beta$ is insufficient to induce significant therapeutic benefits (Fig. 4b, Fig. 8d, e, Fig. 9f). In fact, gene expression analysis of GPF NP-transfected M2-like macrophages revealed a slight shift toward the M1 phenotype (Fig. 3b-d), suggesting that the mere uptake of these neutrally charged particles may induce signals that facilitate M1 polarization.

As expected for a therapeutic approach that does not directly target or lyse tumor cells, IRF5/IKK $\beta$ programming of macrophages delayed tumor progression but did not eradicate the disease in the majority of treated animals. Considering the substantial role TAMs play in cancer proliferation, angiogenesis, invasion, and metastasis ${ }^{44}$, we suspect that our platform could be used to its best advantage as a companion therapeutic for patients that are refractory to other treatments such as immune checkpoint inhibitors, cancer vaccines, $\mathrm{T}$ cell therapies, or antibody approaches.

Our first clinical translation of this technology at Fred Hutchinson Cancer Research Center will be as a monotherapy administered directly via intraperitoneal catheter to treat ovarian cancer patients (as illustrated in Fig. 1). Intraperitoneal chemotherapy, given in cycles over 6-9 weeks, is now standard of care for ovarian cancer patients ${ }^{68,69}$, which means clinical protocols are already in effect on how to best introduce, maintain, and eventually remove the catheter. The advantages to delivering nanoparticles directly into the abdominal cavity in the first Phase
1 trial are: (i) increased safety (limited systemic exposure, and the option to drain and flush the peritoneal cavity as required), (ii) high volume (up to $1 \mathrm{~L}$ of nanoparticles can be safely administered via catheter within minutes), and (iii) direct physical targeting (as ovarian cancer lesions are usually confined within the peritoneal cavity). The results of these investigations will provide important information for the design of Phase 1 trials for systemic applications aimed at treating less-focalized forms of cancer.

\section{Methods}

Cell lines. The murine ovarian cancer cell line ID8, a gift from Dr. Katherine Roby (University of Kansas Medical Center, Kansas City, KS) ${ }^{70}$, was cultured in DMEM supplemented with $4 \%$ FBS and $5 \mu \mathrm{g} / \mathrm{ml}$ insulin, $5 \mu \mathrm{g} / \mathrm{ml}$ transferrin, and $5 \mathrm{ng} / \mathrm{ml}$ sodium selenite (all Sigma-Aldrich). To generate the more aggressive vascular endothelial growth factor (VEGF)-expressing strain, we transfected ID8 tumor cells with the pUNO1 plasmid (Invivogen) encoding murine VEGF and the blasticidinresistance gene. To obtain stable transfectants, tumor cells were cultured in complete medium containing $10 \mu \mathrm{g} / \mathrm{ml}$ Blasticidin (InvivoGen) for three weeks. The THP1-Lucia ${ }^{\mathrm{TM}}$ ISG cells (interferon regulatory factor-inducible reporter monocytes) were purchased from InvivoGen (Cat\# thpl-isg) and cultured in

RPM1-1640 supplemented with $0.05 \mathrm{mM} 2$-mercaptoethanol and $10 \%$ fetal bovine serum (FBS). All cell lines tested negative for mycoplasma based on DNA-based PCR tests (DDC Medical).

PbAE synthesis. We combined 1,4-butanediol diacrylate with 4-amino-1-butanol in a 1:1 molar ratio of diacrylate to amine monomers. Acrylate-terminated poly (4amino-1-butanol-co-1,4-butanediol diacrylate) was formed by heating the mixture to $90^{\circ} \mathrm{C}$ with stirring for $24 \mathrm{~h} .2 .3 \mathrm{~g}$ of this polymer was dissolved in $2 \mathrm{~mL}$ tetrahydrofuran (THF). To form the piperazine-capped 447 polymer, $786 \mathrm{mg}$ of 1-(3aminopropyl)-4-methylpiperazine in $13 \mathrm{~mL}$ THF was added to the polymer/THF solution and stirred at room temperature (RT) for $2 \mathrm{~h}$. The capped polymer was precipitated with 5 volumes of diethyl ether, washed with 2 volumes of fresh ether, and dried under vacuum for 1 day. Neat polymer was dissolved in dimethyl sulfoxide (DMSO) to a concentration of $100 \mathrm{mg} / \mathrm{mL}$ and stored at $-20^{\circ} \mathrm{C}$.

PGA conjugation to Di-mannose. $\alpha$-D-mannopyranosyl-( $1 \rightarrow 2)$ - $\alpha$-D-mannopyranose (Di-mannose, Omicron Biochemicals Inc.) was modified into glycosylamine before being conjugated to PGA. First, the Di-mannose $(157 \mathrm{mg})$ was dissolved in $10.5 \mathrm{~mL}$ of saturated aqueous ammonium carbonate, then stirred at RT for $24 \mathrm{~h}$. On the second day, more solid ammonium carbonate was added until the Dimannose precipitated from the reaction solution. The mixture was stirred until completion, as measured by TLC, followed by lyophilization to remove the excess ammonium carbonate. Complete removal of volatile salt was accomplished by redissolving the solid in methanol. These procedures created an amine on the anomeric carbon for future conjugation with PGA.

To conjugate aminated Di-mannose to PGA, the substrate was dissolved in water to $30 \mathrm{mg} \mathrm{m} / \mathrm{L}$, then sonicated for $10 \mathrm{~min}$ Ethyl-N'-(3-dimethylaminopropyl) carbodiimide. $\mathrm{HCl}$ in water $(4 \mathrm{mg} / \mathrm{mL}, 30$ equiv.) was added with mixing at RT for $4 \mathrm{~min} \mathrm{~N}$-hydroxysulfosuccinimide in water $(30 \mathrm{mg} / \mathrm{mL}, 35$ equiv.) was incubated with the PGA/EDC solution for $1 \mathrm{~min}$ Aminated Di-mannose in phosphatebuffered saline (PBS) was combined with the resulting activated PGA in a 44:1 molar ratio and mixed at RT for $6 \mathrm{~h}$. Excess reagents were removed by dialysis against water for $24 \mathrm{~h}$.

mRNA synthesis. Codon-optimized mRNA for eGFP, IRF5, and IKK (TriLink Biotechnologies) were capped with the Anti-Reverse Cap Analog 3'-O-Me-m7G $\left(5^{\prime}\right) \mathrm{ppp}\left(5^{\prime}\right) \mathrm{G}$ (ARCA), and fully substituted with the modified ribonucleotides pseudouridine $(\Psi)$ and 5-methylcytidine (m5C).

Nanoparticle preparation. IRF5 and IKK $\beta$ mRNAs were combined at a 3:1 (w:w) ratio and diluted to $100 \mu \mathrm{g} / \mathrm{mL}$ in $25 \mathrm{mM}$ sodium acetate $(\mathrm{NaOAc})$ buffer ( $\mathrm{pH}=$ 5.2). $\mathrm{PbEs}$ ) - 447 (PbAE-447) polymer in DMSO (prepared as described above) was diluted from $100 \mu \mathrm{g} / \mu \mathrm{L}$ to $6 \mu \mathrm{g} / \mu \mathrm{L}$, also in NaOAc buffer. To form the nanoparticles, PbAE-447 polymers were added to the mRNA at a ratio of 60:1 (w:w) and vortexed immediately for $15 \mathrm{~s}$ at a medium speed, then the mixture was incubated at RT for 5 min to allow the formation of PbAE-mRNA polyplexes. In the next step, $100 \mu \mathrm{g} / \mathrm{mL}$ PGA/Di-mannose in NaOAc buffer was added to the polyplexes solution, vortexed for $15 \mathrm{~s}$ at medium speed, and incubated for $5 \mathrm{~min}$ at room temperature. In this process, PGA/Di-mannose coated the surfaces of PbAE-mRNA polyplexes to form the final NPs. For longterm storage, $\mathrm{D}$-sucrose $(60 \mathrm{mg} / \mathrm{mL})$ was added to the NP solutions as a cryoprotectant. The nanoparticles were snap-frozen in dry ice, then lyophilized. The dried NPs were stored at $-20^{\circ} \mathrm{C}$ or $-80{ }^{\circ} \mathrm{C}$ until use. For in vivo experiments, lyophilized NPs were re-suspended in water at a 1:20 (w:v) ratio. 
Characterization of nanoparticle size distribution and $\zeta$-potential. The physiochemical properties of NPs (including hydrodynamic radius, polydispersity, $\zeta$-potential, and stability) were characterized using a Zetapals instrument (Brookhaven Instrument Corporation) at $25^{\circ} \mathrm{C}$. To measure the hydrodynamic radius and polydispersity based on dynamic light scattering, NPs were diluted fivefold into $25 \mathrm{mM} \mathrm{NaOAc}(\mathrm{pH}=5.2)$. To measure the $\zeta$-potential, NPs were diluted 10 -fold in $10 \mathrm{mM}$ PBS ( $\mathrm{pH}=7.0)$. To assess the stability of NPs, freshly prepared particles were diluted in $10 \mathrm{mM}$ PBS buffer $(\mathrm{pH}=7.4)$. The hydrodynamic radius and polydispersity of NPs were measured every $10 \mathrm{~min}$ for $5 \mathrm{~h}$, and their sizes and particle concentrations were derived from Particle Tracking Analysis using a Nanosite 300 instrument (Malvern). Freshly made NPs $(25 \mu \mathrm{L}$ containing $0.83 \mu \mathrm{g}$ of mRNA) were deposited on glow discharge-treated 200 mesh carbon/Formvarcoated copper grids. After $30 \mathrm{~s}$, the grids were treated sequentially with $50 \%$ Karnovsky's fixative, $0.1 \mathrm{M}$ cacodylate buffer, $\mathrm{dH}_{2} \mathrm{O}$, then $1 \%(\mathrm{w} / \mathrm{v})$ uranyl acetate. Samples were imaged with a JEOL JEM-1400 transmission electron microscope operating at $120 \mathrm{kV}$ (JEOL USA).

Bone marrow-derived macrophages (BMDMs) and other cell lines. To prepare BMDMs, bone marrow progenitor cells were harvested from mouse femurs following established protocols ${ }^{71}$. These cells were cultured in complete medium [DMEM supplemented with $4.5 \mathrm{~g} / \mathrm{L}$ D-glucose, L-glutamine, $10 \%$ heat-inactivated FBS, $100 \mathrm{U} / \mathrm{mL}$ penicillin and $100 \mu \mathrm{g} / \mathrm{mL}$, Glutamax $50 \mathrm{~mL} / 500 \mathrm{~mL}$, supplemented with $20 \mathrm{ng} / \mathrm{mL}$ M-CSF (Peprotech, cat\#315-02)] at a seeding density of $0.5-1.0 \mathrm{e} 6 / \mathrm{ml}$. Cells were allowed to differentiate into BMDMs ex vivo for 7 days under $5 \% \mathrm{CO}_{2}$ at $37^{\circ} \mathrm{C}$. Next, they were conditioned with macrophage-conditioned medium [macrophage complete medium supplemented with $20 \mathrm{ng} / \mathrm{mL}$ MPLA (Sigma, cat\#L6895) or $20 \mathrm{ng} / \mathrm{mL}$ IL4 (eBioscience, cat\# 34-8041)]. BMDMs were used between 7-21 days ex vivo. The murine ovarian cancer cell line ID8, a gift from Dr. Katherine Roby (University of Kansas Medical Center, Kansas City, KS), was cultured in DMEM supplemented with $10 \%$ FBS, $100 \mathrm{U} / \mathrm{mL}$ penicillin, $5 \mu \mathrm{g} / \mathrm{mL}$ insulin, $5 \mu \mathrm{g} / \mathrm{mL}$ transferrin, and $5 \mathrm{ng} / \mathrm{mL}$ sodium selenite (all Sigma-Aldrich). To generate the more aggressive VEGF-expressing ID8 strain, we transfected ID8 tumor cells with the pUNO1 plasmid (InvivoGen) encoding murine VEGF along with the blasticidin-resistance gene. To obtain stable transfectants, tumor cells were cultured in complete medium containing $10 \mu \mathrm{g} / \mathrm{mL}$ blasticidin (InvivoGen) for 3 weeks. The B16F10 melanoma cell line (American Type Culture Collection) was cultured in complete RPMI 1640 medium with $10 \%$ FBS, $100 \mathrm{U} / \mathrm{mL}$ penicillin, $2 \mathrm{mM} / \mathrm{L}$-glutamine, $1.5 \mathrm{~g} / \mathrm{L}$ sodium bicarbonate, $4.5 \mathrm{~g} / \mathrm{L}$ glucose, $10 \mathrm{mM}$ HEPES, $1.0 \mathrm{mM}$ sodium pyruvate, and $0.05 \mathrm{mM} 2$-mercaptoethanol. For in vivo bioluminescent imaging, both ID8-VEGF and B16F10 cell lines were retrovirally transduced with firefly luciferase. The DF-1 cell line carrying RACS-PDGF $\beta$ or RCAS-cre retrovirus was cultured in complete medium supplemented with $10 \%$ FBS and $100 \mathrm{U} / \mathrm{mL}$ penicillin under $5 \% \mathrm{CO}_{2}$ at $39^{\circ} \mathrm{C}$.

mRNA transfection of BMDMs. One day prior to transfection, BMDMs were reseeded on 24-well plates in macrophage complete medium at a concentration of $250,000 /$ well. Before transfection, the complete medium was replaced with $300 \mu \mathrm{L}$ unsupplemented DMEM. To transfect these cells, NPs containing $2 \mu \mathrm{g}$ mRNA were added into the base medium and co-cultured with the BMDMs at $37^{\circ} \mathrm{C}$. After $1 \mathrm{~h}$, medium containing NPs was removed, and the cells were cultured an additional $24 \mathrm{~h}$ before evaluation of transfection efficiency and cell viability.

\section{Transfection of BMDMs for macrophage signature gene analysis. BMDMs} were reseeded on 24 -well plates in conditioned medium $24 \mathrm{~h}$ prior to transfection, allowing transformation of the cells into their phenotypes. M2-like macrophages were then exposed to either IRF5/IKK $\beta$ NPs carrying 25\% eGFP mRNA as a reporter, or eGFP NPs (control) containing $2 \mu \mathrm{g} \mathrm{mRNA}$, following the transfection protocol described above. After $24 \mathrm{~h}$, the top $10 \%$ of highly transfected BMDMs (as measured by eGFP expression) were sorted at $24 \mathrm{~h}$ after transfection and were rechallenged in low-dose $(10 \mathrm{ng} / \mathrm{mL})$ IL4 medium for another $48 \mathrm{~h}$ before RNA isolation. RNAs extracted from these cells were compared to those from standard M1- or M2-like macrophages so we could identify signature genes associated with IRF5-NP treatment.

RNA isolation and preparation. To harvest RNAs, BMDMs were lysed in Trizol reagent (Ambion), and total RNAs were extracted and purified using RNeasy ${ }^{\circledR}$ Plus Universal Mini-Kits (QIAGEN) following the manufacturer's instructions. Sample RNA was quantified using a NanoDrop Microvolume Spectrophotometer (Thermo Fisher) and then subjected to quality control performed by the FHCRC Genomics Shared Resource with an Agilent 4200 TapeStation analyzer (Agilent).

IRF5 protein ELISA. Following nanoparticle transfection, macrophage cell pellets were subjected to total protein extraction using RIPA Lysis and Extraction Buffer supplemented with Halt Protease Inhibitor Cocktail and Halt Phosphatase Inhibitor Cocktail (Thermo Fisher Scientific), following the manufacturer's protocol. After the protein concentration in each sample was quantified via a BCA assay, the IRF5 concentration from each sample was evaluated using the ELISA assay (LSF21481, LSBio, WA). The IRF5 concentrations measured by ELSA were normalized by the total protein concentration.
Macrophage signature gene analysis using NanoString technology. Gene expression values from stimulated BMDM cultures were measured using the nCounter ${ }^{\oplus}$ Myeloid Innate Immunity Panel (NanoString Technology), which analyzes 770 genes occurring in 19 different pathways and processes them across seven different myeloid cell types. The samples were tested using an nCounter Analysis System (NanoString Technologies). Raw data were processed and checked for quality using the R/Bioconductor NanoStringQCPro software package ${ }^{72}$. Expression values were normalized to the geometric mean of housekeeping genes and log2-transformed using nSolver 4.0 software (NanoString Technologies). False Discovery Rates for ratio data were calculated from the $p$-values returned by the $t$ tests using the Benjamini-Yekutieli method.

Flow cytometry and cell sorting. Cells obtained from spleen, blood, peritoneal lavage, and bronchoalveolar lavage were analyzed by flow cytometry with myeloid and lymphoid immunophenotyping panels using the anti-mouse antibody probes listed in Supplementary Table 1. Primary antibodies applied in the flow cytometry analysis are listed as follows: CD45 (eBioscience catalog \# 48-0451, 1:800), MHC IA/I-E (Biolegend catalog \# 107622, 1:400), CD11b (BD Biosciences catalog \# $557657,1: 200$ ), CD11c (BD Biosciences catalog \# 5624547, 1:200), Ly6C (eBioscience catalog \# 45-4932, 1:200), Ly6G (Biolegend catalog \# 127624, 1:200), CD206 (Biolegend catalog \# 141732, 1:200), CD335 (Nkp46) (BD Biosciences catalog \# 565085, 1:800), CD4 (Biolegend catalog \# 100540, 1:400), CD44 (BD Biosciences catalog \# 562464, 1:400), CD49B (BD Biosciences catalog \# 740250, 1:200), CD62L (Biolegend catalog \# 104428, 1:200), CD8 (Biolegend catalog \# $100712,1: 400$ ), TCR- $\beta$ chain (Biolegend catalog \# 127908, 1:400), F4/80 (eBioscience catalog \# 12-4801, 1:400). Data were collected using a BD LSRFortessa analyzer running FACSDIVA software (Beckton Dickinson). CD11b+ and F4/80+ peritoneal macrophages were sorted using BD FACS ARIA II. All collected data were analyzed using FlowJo 10.0 software.

Cytokine analysis. Cytokine levels were evaluated using a Luminex 200 system (Luminex) at the FHCRC Immune Monitoring Shared Resource center. For ex vivo studies, cell culture supernatant was collected for the measurement of IL-6, IL$12 \mathrm{p} 70$, INF $\gamma$, and TNFa concentrations. For in vivo studies, plasma concentration of GM-CSF, INF $\gamma$, IL-12p70, IL-2, IL-6, and TNF $\alpha$ were measured. IL-1 $\beta$ cytokine levels were measured using the Invitrogen ELISA kit (REF\#88-7013)

Histology. Immunofluorescence, immunohistochemistry (IHC), and hematoxylin and eosin (H\&E) analysis were performed on mouse intestinal mesentery tissue. For all histopathology analyses, tissues were fixed in $4 \%$ neutral buffer formalin before further processing. Four-micron sections were cut and stained with the Leica Bond Rx (Leica Biosystems, Buffalo Grove, IL). For IHC DAB staining, slides were pretreated with Leica Bond Epitope Retrieval Solution for 20 min Endogenous peroxidase was blocked with Leica peroxide block for 5 min A TCT protein block was applied for $10 \mathrm{~min}(0.05 \mathrm{M}$ Tris, $0.15 \mathrm{M} \mathrm{NaCl}, 0.25 \%$ Casein, $0.1 \%$ Tween 20 $\mathrm{pH}$ 7.6). Primary antibody was applied to the tissue for $60 \mathrm{~min}$ The antibody was then detected using a specific polymer and staining was visualized with Bond Polymer Refine Detection DAB (Leica Biosystems catalog\# D59800); a hematoxylin counterstain was also used (Leica). Primary antibodies applied in the immunofluorescence and IHC DAB analysis are listed as follows: Ly6B.2 (Bio-Rad catalog \# MCA771GA, 1:7500), F4/80 (Cell Signaling catalog \# 770765, 1:6000), CD4 (eBioscience catalog \# 14-976-32, 1:4000), CD8 (eBioscience catalog \# 14-0808-82, 1:4000), Cytokeratin 8/18 (University of Iowa Hybridoma Bank: catalog \# TROMA-1, 1:100). DAPI (Sigma Catalog 8417-10MG) was used at $5 \mu \mathrm{g} / \mathrm{ml}$ in PBS.

A Perkin Elmer Vectra 3.0 Automated Imaging Platform was used to acquire the fluorescence images of intestinal mesentery slides under $\times 20$ magnification. These images were analyzed using HALO Image Analysis Modules. The Cytonuclear for FL function was applied to calculate \%CD4+ cells, \%CD8+ cells and $\% \mathrm{~F} 4 / 80+\%$ Ly6B. $2+$ cells among all cells in the tumor region. For each sample group, five tissue slides prepared from three mice were analyzed to obtain statistically significant data.

qRT-PCR analysis. Gene expression levels were determined by qRT-PCR. To measure selected macrophage signature genes (SerpinB2, Retnla, Ccl5, Ccl11, codon-optimized IRF5, endogenous IRF5, and housekeeping GAPD genes), total RNA was isolated with RNeasy mini-columns (Qiagen) according to the manufacturer's instructions. cDNA was synthesized using a qScript cDNA Synthesis Kit (Quanta). For each sample, qRT-PCR was performed in triplicate via PerfeCTa qPCR SuperMix Low ROX (Quanta) using gene-specific probes from the Roche's Universal Probe Library (UPL) and PCR primers optimized by ProbeFinder (Roche): SerpinB2, UPL -049, F-ACTGGGGCAGTTATGACAGG, R-GATGA TCGGCCACAAACTG; Retnla, UPL-078, F-TTGTTCCCTTCTCATCTGCAT, RCCTTGACCTTATTCTCCACGA; Ccl5, UPL-105, F-CCTACTCCCACTC GGTCCT, R-CTGATTTCTTGGGTTTGCTGT; Ccl11, UPL-018, F-AGAGCTC CACAGCGCTTC, R- CAGCACCTGGGAGGTGAA; codon-optimized IRF5, UPL-022, F-TCTTAAAGACCACATGGTAGAACAGT, R-AGCTGCTGTTGGG ATTGC; endogenous IRF5, UPL-011, F-GCTGTGCCCTTAACAAAAGC, R-GGC TGAGGTGGCA TGTCT. Signature gene mRNA levels were normalized based on 
amplification of GAPD, UPL-060, F-AGCCACATCGCTCAGACAC and RGCCCAATACGACCAAATCC. All qRT-PCR reactions were performed using Quant Studio5 RT-PCR machines running QuantStudio6 software (Applied Biosystems). In cases when the amplification plot did not cross the threshold and no Ct value was obtained ("undetermined"), a Ct value equal to the highest cycle number of in the assay ( 40 cycles) was used for comparisons of relative expression.

Mice and in vivo tumor models. Except for the brain tumor model-related experiments, the mice used in these experiments were obtained from Jackson Laboratory; the others were bred and housed in the FHCRC animal facility. All of the mice were used in the context of a protocol approved by the center's Institutional Animal Care and Use Committee. To model ovarian tumors, $5 \times 10^{6}$ vascular epithelial growth factor (VEGFP)-expressing ID8 cells were injected intraperitoneally (i.p.) into 4- to 6-week-old female albino B6 (C57BL/6J-Tyr $<\mathrm{c}-2 \mathrm{~J}>)$ mice and allowed to establish for 2 weeks. For survival studies, the animals were treated i.p. with IRF5 NPs/eGFP NPs carrying $50 \mu \mathrm{g}$ mRNA (two doses per week for 9 weeks, or until health conditions reached euthanizing requirements). For mechanism studies, we used the treatments for either 1, 2, or 3 weeks, followed by euthanization at $48 \mathrm{~h}$ following the last dose. Peritoneal lavage was performed to collect the peritoneal cells. To compare the efficacy of IRF5/IKK $\beta$ NPs with status quo macrophage targeting therapies, one group of mice received treatment with IRF5/IKK $\beta$ NPs carrying $50 \mu \mathrm{g}$ mRNA for 3 weeks with 2 doses per week; the second received oral gavage of $15 \mathrm{mg} / \mathrm{kg}$ PI3K $\gamma$ inhibitor IPI-594 (MedKoo Biosciences Inc) formulated in vehicle (5\% 1-methyl-2-pyrrolidinone in polyethylene glycol 400) daily for 3 weeks; the third group received i.p. injection of $30 \mathrm{mg} / \mathrm{kg}$ CSF1R inhibitor Pexidartinib (PLX3397, MedKoo Biosciences Inc) formulated in the same vehicle daily for 3 weeks.

To model metastatic lung cancer, $2.5 \times 10^{4} \mathrm{~B} 16 \mathrm{~F} 10$ cells transduced with F-luc and suspended in $200 \mu \mathrm{L}$ RPMI medium were injected into 4 - to 6-week-old female albino B6 (C57BL/6J-Tyr $<\mathrm{c}-2 \mathrm{~J}>$ ) mice (Jackson Laboratories) and allowed to establish for 1 week. For survival studies, mice were treated retro-orbitally with (or without) IRF5/IKK $\beta$ or eGFP NPs carrying $30 \mu \mathrm{g}$ mRNA suspended in PBS. Mice were treated with 3 doses/wk for 3 weeks or until health conditions reached euthanizing requirements. For mechanism studies, the mice received the same treatments for 2 weeks. Bronchoalveolar lavage was performed to collect alveolar cells for analysis.

Mice bearing glioma were generated following published protocols ${ }^{49}$. Avian DF1 cells producing RCAS-PDGF $\beta$ and RCAS-cre retroviruses were injected intracranially into both brain hemispheres (coordinates: $1 \mathrm{~mm}$ caudal from bregma, $2 \mathrm{~mm}$ lateral, depth of $2 \mathrm{~mm}$ from the dural surface) of Nestin-tv-a/Ink4aarf-/-; Pten-/ - mice (C57BL/6) between 4-6 weeks of age. Tumors were allowed to establish for 2 weeks. At day 15, mice received 10Gy radiation to one hemisphere, while the unirradiated hemisphere was shielded with lead. The next day, mice received retro-orbital injections of IRF5/IKK $\beta$ NPs carrying $30 \mu \mathrm{g}$ mRNA (3 doses/wk for 3 weeks), or were assigned to the PBS control group.

In vivo bioluminescence imaging. D-Luciferin (Xenogen) in PBS (15 mg/mL) was used as a substrate for firefly luciferase imaging. Bioluminescence images were collected with a Xenogen IVIS Spectrum Imaging System (Xenogen). Mice were anesthetized with $2 \%$ isoflurane (Forane, Baxter Healthcare) before and during imaging. For ID8-VEGF ovarian tumors, each mouse was injected i.p. with $300 \mu \mathrm{g}$ of D-Luciferin, and images were collected 10 min later. For B16F10 lung metastatic tumors, mice were injected i.p. with $3 \mathrm{mg}$ of D-Luciferin, and images were collected $15 \mathrm{~min}$ afterwards. For brain tumor models, the mice received retro-orbital injection of $75 \mathrm{mg} / \mathrm{kg}$ body weight D-Luciferin, and images were collected $4 \mathrm{~min}$ later. Acquisition times ranged from $10 \mathrm{~s}$ to $5 \mathrm{~min}$.

Biodistribution analysis. To determine the biodistribution of IRF5 NPs in the ID8-VEGF ovarian tumor model, mice in 7-8 groups received an i.p. or retroorbital dose of NPs carrying $50 \mu \mathrm{g}$ mRNA. Twenty-four hours after injection, whole blood was collected and mice were euthanized with $\mathrm{CO}_{2}$ to retrieve organs (liver, spleen, lung, kidney, heart, intestine, pancreases, and diaphragm). All tissues were stabilized with RNAlater, then frozen on dry ice. The codon-optimized IRF5 mRNA levels in each organ were measured using RT-qPCR.

Toxicity analysis. To measure potential in vivo toxicities of repeatedly injecting macrophage-targeting NPs, we injected mice (5/group) intraperitoneally or intravenously with six sequential doses of IRF5/IKK $\beta$ or eGFP mRNA NPs. Controls received no treatment. Twenty-four hours after the final infusion, mice were anesthetized and blood was collected by retro-orbital bleed to determine the complete blood counts. Blood was also collected for serum chemistry and cytokine profile analyses (performed by Phoenix Central Laboratories, Mukilteo, WA). Animals were then euthanized with $\mathrm{CO}_{2}$ to retrieve organs, which were washed with deionized water before fixation in $4 \%$ paraformaldehyde. The tissues were processed routinely, and sections were stained with H\&E. The specimens were interpreted by board-certified staff pathologists, in a blinded fashion.

Cytokine assays. Cytokine levels were evaluated using a Luminex 200 system (Luminex) at the FHCRC Immune Monitoring Shared Resources. For ex vivo studies, cell culture supernatant was collected for the measurement of IL-6, IL12p70, INF $\gamma$, and TNFa concentrations. For in vivo studies, we measured plasma concentrations of IL-6, TNFa, and IL- $1 \beta$.

Statistical analysis. The statistical significance of observed differences were analyzed using the unpaired, two-tailed Student's $t$ test or the unpaired, two-tailed one-way ANOVA test. The $P$ values for each measurement are listed in the figures or figure legends. We characterized survival data using the Log-rank test. All statistical analyses were performed either using GraphPad Prism software version 6.0 or $\mathrm{R}$ software.

Study approval. The care and use of mice in this study was approved by the Institutional Animal Care \& Use Committee (IACUC) at the Fred Hutchinson Cancer Research Center, and was in compliance with all relevant ethical regulations for animal testing and research (Assurance \#A3226-01, IACUC Protocol Number 50782).

Reporting summary. Further information on research design is available in the Nature Research Reporting Summary linked to this article.

\section{Data availability}

The Raw and processed data from the NanoString gene expression assays data have been deposited in the NCBI's Gene Expression Omnibus database under the accession code GSE120254 and GSE129498. All the other data supporting the findings of this study are available within the article and its supplementary information files and directly from M. Stephan upon reasonable request. A reporting summary for this article is available as a Supplementary Information file.

Received: 9 October 2018 Accepted: 9 August 2019

Published online: 03 September 2019

\section{References}

1. Yang, L. \& Zhang, Y. Tumor-associated macrophages: from basic research to clinical application. J. Hematol. Oncol. 10, 58 (2017).

2. Murray, P. J. Macrophage polarization. Annu Rev. Physiol. 79, 541-566 (2017).

3. Murray, P. J. et al. Macrophage Activation and Polarization: Nomenclature and Experimental Guidelines. Immunity 41, 14-20 (2014).

4. Jarosz-Biej, M. et al. M1-like macrophages change tumor blood vessels and microenvironment in murine melanoma. PLoS ONE 13, e0191012 (2018).

5. Yuan, A. et al. Opposite effects of M1 and M2 macrophage subtypes on lung cancer progression. Sci. Rep. 5, 14273 (2015).

6. Caux, C., Ramos, R. N., Prendergast, G. C., Bendriss-Vermare, N. \& Menetrier-Caux, C. A milestone review on how macrophages affect tumor growth. Cancer Res 76, 6439-6442 (2016).

7. Chen, Y. L., Zhang, S. Y., Wang, Q. Z. \& Zhang, X. B. Tumor-recruited M2 macrophages promote gastric and breast cancer metastasis via M2 macrophage-secreted CHI3L1 protein. J. Hematol. Oncol. 10, 36 (2017).

8. Sousa, S. et al. Human breast cancer cells educate macrophages toward the M2 activation status. Breast Cancer Res. 17, 101 (2015).

9. De Palma, M. \& Lewis, C. E. Cancer: macrophages limit chemotherapy. Nature 472, 303-304 (2011).

10. Mantovani, A., Marchesi, F., Malesci, A., Laghi, L. \& Allavena, P. Tumourassociated macrophages as treatment targets in oncology. Nat. Rev. Clin. Oncol. 14, 399-416 (2017).

11. Mantovani, A. et al. The chemokine system in diverse forms of macrophage activation and polarization. Trends Immunol. 25, 677-686 (2004).

12. Muller, E. et al. Toll-like receptor ligands and interferon-gamma synergize for induction of antitumor M1 macrophages. Front. Immunol. 8, 1383(2017).

13. Wiehagen, K. R. et al. Combination of CD40 agonism and CSF-1R blockade reconditions tumor-associated macrophages and drives potent antitumor immunity. Cancer Immunol. Res. 5, 1109-1121 (2017).

14. Sangro, B. et al. Phase I trial of intratumoral injection of an adenovirus encoding interleukin-12 for advanced digestive tumors. J. Clin. Oncol. 22, 1389-1397 (2004)

15. Vonderheide, R. H. et al. Phase I study of the CD40 agonist antibody CP870,893 combined with carboplatin and paclitaxel in patients with advanced solid tumors. Oncoimmunology 2, e23033 (2013).

16. Pockros, P. J. et al. Oral resiquimod in chronic HCV infection: safety and efficacy in 2 placebo-controlled, double-blind phase IIa studies. J. Hepatol. 47, 174-182 (2007)

17. Pyonteck, S. M. et al. CSF-1R inhibition alters macrophage polarization and blocks glioma progression. Nat. Med. 19, 1264-1272 (2013). 
18. Tap, W. D. et al. Structure-guided blockade of CSF1R kinase in tenosynovial giant-cell tumor. N. Engl. J. Med. 373, 428-437 (2015).

19. Nywening, T. M. et al. Targeting tumour-associated macrophages with CCR2 inhibition in combination with FOLFIRINOX in patients with borderline resectable and locally advanced pancreatic cancer: a single-centre, open-label, dose-finding, non-randomised, phase $1 \mathrm{~b}$ trial. Lancet Oncol. 17, 651-662 (2016).

20. Butowski, N. et al. Orally administered colony stimulating factor 1 receptor inhibitor PLX3397 in recurrent glioblastoma: an Ivy Foundation Early Phase Clinical Trials Consortium phase II study. Neuro Oncol. 18, 557-564 (2016).

21. Sauter, K. A. et al. Pleiotropic effects of extended blockade of CSF1R signaling in adult mice. J. Leukoc. Biol. 96, 265-274 (2014).

22. Fritz, J. M. et al. Depletion of tumor-associated macrophages slows the growth of chemically induced mouse lung adenocarcinomas. Front Immunol. 5, 587 (2014).

23. Chao, M. P., Weissman, I. L. \& Majeti, R. The CD47-SIRPalpha pathway in cancer immune evasion and potential therapeutic implications. Curr. Opin. Immunol. 24, 225-232 (2012).

24. Azad, A. K., Rajaram, M. V. \& Schlesinger, L. S. Exploitation of the macrophage mannose receptor (CD206) in infectious disease diagnostics and therapeutics. J. Cytol. Mol. Biol. 1, 1000003 (2014).

25. Mazzieri, R. et al. Targeting the ANG2/TIE2 axis inhibits tumor growth and metastasis by impairing angiogenesis and disabling rebounds of proangiogenic myeloid cells. Cancer Cell 19, 512-526 (2011).

26. Nuhn, L. et al. Targeting protumoral tumor-associated macrophages with nanobody-functionalized nanogels through strain promoted azide alkyne cycloaddition ligation. Bioconjug. Chem. 29, 2394-2405 (2018).

27. Zhu, S. J., Niu, M. M., O'Mary, H. \& Cui, Z. R. Targeting of tumor-associated macrophages made possible by PEG-sheddable, mannose-modified nanoparticles. Mol. Pharm. 10, 3525-3530 (2013).

28. Song, M. L., Liu, T., Shi, C. R., Zhang, X. Z. \& Chen, X. Y. Bioconjugated manganese dioxide nanoparticles enhance chemotherapy response by priming tumor-associated tL.A macrophages toward M1-like phenotype and 11 attenuating tumor hypoxia (vol 10, pg 633, 2016). Acs Nano 10, 3872-3872 (2016).

29. Kwon, H. et al. Emergence of synthetic mRNA: in vitro synthesis of mRNA and its applications in regenerative medicine. Biomaterials 156, 172-193 (2018).

30. Fuchs, A. L., Neu, A. \& Sprangers, R. A general method for rapid and costefficient large-scale production of 5 ' capped RNA. RNA 22, 1454-1466 (2016).

31. Jemielity, J. et al. Novel "anti-reverse" cap analogs with superior translational properties. RNA 9, 1108-1122 (2003).

32. Kariko, K. et al. Incorporation of pseudouridine into mRNA yields superior nonimmunogenic vector with increased translational capacity and biological stability. Mol. Ther. 16, 1833-1840 (2008).

33. Kormann, M. S. et al. Expression of therapeutic proteins after delivery of chemically modified mRNA in mice. Nat. Biotechnol. 29, 154-157 (2011).

34. Pastor, F. et al. An RNA toolbox for cancer immunotherapy. Nat. Rev. Drug Disco. 17, 751-767 (2018).

35. Zheng, Y. H. et al. Effect of bone marrow mesenchymal stem cells on the polarization of macrophages. Mol. Med. Rep. 17, 4449-4459 (2018).

36. Krausgruber, T. et al. IRF5 promotes inflammatory macrophage polarization and TH1-TH17 responses. Nat. Immunol. 12, 231-238 (2011).

37. Ren, J., Chen, X. \& Chen, Z. J. IKKbeta is an IRF5 kinase that instigates inflammation. Proc. Natl Acad. Sci. USA 111, 17438-17443 (2014).

38. Quabius, E. S. \& Krupp, G. Synthetic mRNAs for manipulating cellular phenotypes: an overview. N. Biotechnol. 32, 229-235 (2015).

39. Smith, T. T. et al. In situ programming of leukaemia-specific T cells using synthetic DNA nanocarriers. Nat. Nanotechnol. 12, 813-820 (2017).

40. Tarrant, J. M. Blood cytokines as biomarkers of in vivo toxicity in preclinical safety assessment: considerations for their use. Toxicol. Sci. 117, 4-16 (2010).

41. Copeland, S. et al. Acute inflammatory response to endotoxin in mice and humans. Clin. Diagn. Lab Immunol. 12, 60-67 (2005).

42. Ju, C. \& Tacke, F. Hepatic macrophages in homeostasis and liver diseases: from pathogenesis to novel therapeutic strategies. Cell Mol. Immunol. 13, 316-327 (2016).

43. Butler, K. L., Clancy-Thompson, E. \& Mullins, D. W. CXCR3(+) monocytes/ macrophages are required for establishment of pulmonary metastases. Sci. Rep. 7, 45593 (2017).

44. Nielsen, S. R. \& Schmid, M. C. Macrophages as key drivers of cancer progression and metastasis. Mediat. Inflamm. 2017, 9624760 (2017).

45. Hambardzumyan, D., Gutmann, D. H. \& Kettenmann, H. The role of microglia and macrophages in glioma maintenance and progression. Nat. Neurosci. 19, 20-27 (2016)
46. Mann, J., Ramakrishna, R., Magge, R. \& Wernicke, A. G. Advances in radiotherapy for glioblastoma. Front. Neurol. 8, 748 (2017).

47. Hambardzumyan, D., Amankulor, N. M., Helmy, K. Y., Becher, O. J. \& Holland, E. C. Modeling Adult Gliomas Using RCAS/t-va Technology. Transl. Oncol. 2, 89-95 (2009).

48. Quail, D. F. et al. The tumor microenvironment underlies acquired resistance to CSF-1R inhibition in gliomas. Science 352, aad3018 (2016).

49. Uhrbom, L., Nerio, E. \& Holland, E. C. Dissecting tumor maintenance requirements using bioluminescence imaging of cell proliferation in a mouse glioma model. Nat. Med. 10, 1257-1260 (2004).

50. Li, C., Levin, M. \& Kaplan, D. L. Bioelectric modulation of macrophage polarization. Sci. Rep. 6, 21044 (2016).

51. Surdziel, E. et al. Multidimensional pooled shRNA screens in human THP-1 cells identify candidate modulators of macrophage polarization. PLOS ONE 12, e0183679 (2017).

52. Pardi, N., Hogan, M. J., Porter, F. W. \& Weissman, D. mRNA vaccines - a new era in vaccinology. Nat. Rev. Drug Disco. 17, 261-279 (2018).

53. Ren, J. et al. Multiplex genome editing to generate universal CAR T cells resistant to PD1 inhibition. Clin. Cancer Res. 23, 2255-2266 (2017).

54. Hale, M. et al. Homology-directed recombination for enhanced engineering of chimeric antigen receptor T cells. Mol. Ther. Methods Clin. Dev. 4, 192-203 (2017).

55. Steinle, H., Behring, A., Schlensak, C., Wendel, H. P. \& Avci-Adali, M. Concise review: application of in vitro transcribed messenger RNA for cellular engineering and reprogramming: progress and challenges. Stem Cells 35, 68-79 (2017).

56. Weiss, M., Blazek, K., Byrne, A. J., Perocheau, D. P. \& Udalova, I. A. IRF5 is a specific marker of inflammatory macrophages in vivo. Mediat. Inflamm. 2013, 245804 (2013).

57. Ramirez-Carvajal, L. et al. Expression of porcine fusion protein IRF7/3(5D) efficiently controls foot-and-mouth disease virus replication. J. Virol. 88, 11140-11153 (2014).

58. Chang, T. H., Xu, S., Tailor, P., Kanno, T. \& Ozato, K. The small ubiquitin-like modifier-deconjugating enzyme sentrin-specific peptidase 1 switches IFN regulatory factor 8 from a repressor to an activator during macrophage activation. J. Immunol. 189, 3548-3556 (2012).

59. Chistiakov, D. A., Myasoedova, V. A., Revin, V. V., Orekhov, A. N. \& Bobryshev, Y. V. The impact of interferon-regulatory factors to macrophage differentiation and polarization into M1 and M2. Immunobiology 223, 101-111 (2018).

60. Navegantes, K. C. et al. Immune modulation of some autoimmune diseases: the critical role of macrophages and neutrophils in the innate and adaptive immunity. J. Transl. Med. 15, 36 (2017).

61. Anderson, B. R. et al. Incorporation of pseudouridine into mRNA enhances translation by diminishing PKR activation. Nucleic Acids Res. 38, 5884-5892 (2010).

62. Pardi, N. et al. Nucleoside-modified mRNA vaccines induce potent $\mathrm{T}$ follicular helper and germinal center B cell responses. J. Exp. Med. 215, 1571-1588 (2018).

63. Andries, O. et al. N(1)-methylpseudouridine-incorporated mRNA outperforms pseudouridine-incorporated mRNA by providing enhanced protein expression and reduced immunogenicity in mammalian cell lines and mice. J. Control Release 217, 337-344 (2015).

64. Schallon, A., Synatschke, C. V., Jerome, V., Muller, A. H. \& Freitag, R. Nanoparticulate nonviral agent for the effective delivery of pDNA and siRNA to differentiated cells and primary human T lymphocytes. Biomacromolecules 13, 3463-3474 (2012).

65. Chen, G. et al. MRI-visible polymeric vector bearing CD3 single chain antibody for gene delivery to $\mathrm{T}$ cells for immunosuppression. Biomaterials $\mathbf{3 0}$, 1962-1970 (2009).

66. Liu, J., Stace-Naughton, A., Jiang, X. \& Brinker, C. J. Porous nanoparticle supported lipid bilayers (protocells) as delivery vehicles. J. Am. Chem. Soc. 131, 1354-1355 (2009).

67. Mangraviti, A. et al. Polymeric nanoparticles for nonviral gene therapy extend brain tumor survival in vivo. ACS Nano 9, 1236-1249 (2015).

68. Jewell, A., McMahon, M. \& Khabele, D. Heated intraperitoneal chemotherapy in the management of advanced ovarian cancer. Cancers 10, 296. https://doi. org/10.3390/cancers100090296 (2018).

69. Robella, M. et al. Treatment of peritoneal carcinomatosis from ovarian cancer by surgical cytoreduction and hyperthermic intraperitoneal chemotherapy (HIPEC). Minerva Chir. 69, 27-35 (2014).

70. Roby, K. F. et al. Development of a syngeneic mouse model for events related to ovarian cancer. Carcinogenesis 21, 585-591 (2000).

71. Zhang, X., Goncalves, R. \& Mosser, D. M. The isolation and characterization of murine macrophages. Curr. Protoc. Immunol. Chapter 14, Unit 1411 (2008). 
72. Nickles, D., Sandmann, T., Ziman, R., \& Bourgon, R. NanoStringQCPro: Quality metrics and data processing methods for NanoString mRNA gene expression data. R package version 1.10 .0 (2018).

\section{Acknowledgements}

We thank the FHCRC Genomics Core staff for assistance in the preparation and analysis of NanoString gene expression assays. This work was supported in part by the FHCRC Immunotherapy Initiative with funds provided by the Bezos Family Foundation, by the Experimental Histopathology Shared Resource of the Fred Hutchinson/University of Washington Cancer Consortium (P30 CA015704), and by the National Science Foundation (CAREER Award \#1452492 and EAGER Award \#1644363). M.T.S. was also supported by a Research Scholar Grant (RSG-16-110-01- LIB) from the American Cancer Society. F.Z. received a Basic Research Fellowship from the American Brain Tumor Association (ABTA).

\section{Author contributions}

F.Z. and N.N.P. helped conceive the study, and designed and performed the experiments. C.I.E induced gliomas in mice. A.L.K. performed and analyzed in vivo safety/toxicity studies. S.B.S. produced polymers and Di-mannose conjugates. M.E.C. performed NanoString gene expression assays. E.C.H. developed and provided genetically engineered mouse models of brain cancer, and M.T.S. conceived the study, helped designed the experiments, and wrote the manuscript.

\section{Additional information}

Supplementary Information accompanies this paper at https://doi.org/10.1038/s41467019-11911-5.
Competing interests: M.T.S. is a consultant of Tidal Therapeutics (Cambridge, MA) and holds stocks in the company. The remaining authors declare no competing interest.

Reprints and permission information is available online at http://npg.nature.com/ reprintsandpermissions/

Peer review information: Nature Communications thanks Mansoor Amiji and Jo Van Ginderachter for their contribution to the peer review of this work.

Publisher's note: Springer Nature remains neutral with regard to jurisdictional claims in published maps and institutional affiliations.

(c) (i) Open Access This article is licensed under a Creative Commons Attribution 4.0 International License, which permits use, sharing, adaptation, distribution and reproduction in any medium or format, as long as you give appropriate credit to the original author(s) and the source, provide a link to the Creative Commons license, and indicate if changes were made. The images or other third party material in this article are included in the article's Creative Commons license, unless indicated otherwise in a credit line to the material. If material is not included in the article's Creative Commons license and your intended use is not permitted by statutory regulation or exceeds the permitted use, you will need to obtain permission directly from the copyright holder. To view a copy of this license, visit http://creativecommons.org/ licenses/by/4.0/.

(C) The Author(s) 2019 